\title{
Computational chemo-thermo-mechanical coupling phase-field model for complex fracture induced by early-age shrinkage and hydration heat in cement-based materials
}

\author{
Thanh-Tung Nguyen ${ }^{a, *}$, Danièle Waldmanna ${ }^{a}$, Tinh Quoc Bui ${ }^{b}$ \\ a University of Luxembourg, Laboratory of Solid Structures, 6, Rue Richard Coudenhove-Kalergi, L-1359, Luxembourg \\ ${ }^{\mathrm{b}}$ Department of Civil and Environmental Engineering, Tokyo Institute of Technology, 2-12- 1-W8- 22, Ookayama, \\ Meguro-ku, Tokyo 152-8552, Japan
}

Received 28 February 2018; received in revised form 26 October 2018; accepted 8 January 2019

Available online 22 January 2019

\begin{abstract}
In this paper, we present a new multi-physics computational framework that enables us to capture and investigate complex fracture behavior in cement-based materials at early-age. The present model consists of coupling the most important chemothermo-mechanical processes to describe temperature evolution, variation of hydration degree, and mechanical behavior. The changes of material properties are expressed as a function of the hydration degree, to capture the age effects. Fracture analysis of these processes is then accommodated by a versatile phase field model in the framework of smeared crack models, addressing the influence of cracks on hydration and thermal transfer. We additionally describe a stable and robust numerical algorithm, which aims to solve coupled problems by using a staggered scheme. The developed approach is applied to study the fracture phenomena for both homogeneous and heterogeneous concrete structures. Especially, in the second case, all microstructural heterogeneities of sand and cement matrix are explicitly accounted. Nucleation, initiation, and propagation of complex crack network are simulated in an efficient way demonstrating the potential of the proposed approach to assess the early-age defects in concrete structures and materials.
\end{abstract}

(C) 2019 Elsevier B.V. All rights reserved.

Keywords: Early-age cracking; Cement hydration; Fracture; Phase field model; FEM

\section{Introduction}

Early-age shrinkage and hydration heat induced defects in concrete have been a major research area in the past few decades. They have been determined as important and attractive problems in computational mechanics due to their strong effects on the life span of structures. Since the change of mechanical properties of early-age concrete is fast, and is largely dependent on the physical and chemical processes (i.e., the hydration), the modeling of complex fracture in such cement-based materials at early-age stage by means of numerical approaches is generally thus not a trivial task. The occurrence of early-age cracking also causes several serious problems, such as waterproof

\footnotetext{
* Corresponding author.

E-mail address: thanh-tung.nguyen@uni.lu (T.-T. Nguyen).
} 
requirement, damage of structural integrity, exposition to chemical attack, especially in the marine environments and nuclear reactor containments. Developing effective numerical models, also the scope of the present work, for accurately interpreting fracture resistance of the cement-based materials at early-age stage after casting in terms of key engineering parameters is highly required.

The hydration processes during hardening of young concrete often lead to a considerable volume change and thermal deformation. At that period, the strength of concrete (i.e., stiffness and fracture resistance) is still low, so that the thermal and shrinkage strains may induce premature damage/crack in the structure. Due to the complexity of the hydration process, including many other aspects, such as chemical, thermal, mechanical, and environmental effects, numerical simulation of crack nucleation and propagation, from microscopic to structural length-scales, remains a challenging and largely open issue.

Several numerical methods have been proposed in the literature to study cracks induced by the shrinkage and hydration heat. For instance, de Borst and Van den Boogaard [1] presented a computational approach for fracture assessment without considering micro-level behavior. In the work of Song et al. [2], the authors introduced an analytical method to evaluate the evolution of material properties during the hydration process. The crack growth is then described by using finite element analysis (FEA). A theoretical procedure, based on the characteristics of concrete at both material and micromechanical levels, has been developed in Yuan and Wan [3], which was to simulate the whole process after concrete setting. The phenomena of crack initiation and propagation due to the cement hydration were also investigated and reported in Schutter [4], wherein a new model is proposed by introducing a degree of hydration, which was then applied to evaluate the hardening process of massive concrete elements. Another interesting approach was presented in Bažant et al. [5], they reported a new method based on crack band model with smeared cracking, considering creep strain, and taking into account the aging and temperature effects on fracture behavior in massive concrete wall. A rare work mentioned microstructure effects was developed by Grassl et al. [6]. In that study, the micro-cracking induced by shrinkage is simulated by using the lattice approach, exploring the effects of aggregate size and volume fraction on fracture behavior of mortar and concrete. Recently, the early-age behavior of massive concrete was analyzed by a numerical model of coupling chemo-thermal and elastic-damage model Briffaut et al. [7], in which the Mazars's damage criterion [8] is adopted for their fracture model. Some other contributions in this field can be found e.g., in [9-15]. However, those studies are based on a simple model of fracture mechanics and often neglect the influences of crack on cement hydration and thermal transfer. An effective computational approach that enables us to model complex crack initiation, coalesce, and propagation of cement-based materials induced by hydration processes, and takes into account, for instance, thermal deformation effect, is still missing.

In recent years, a variational numerical technique, the phase field method, has been introduced based on the pioneer work of Marigo and Francfort [16], allowing the development of fracture models at different scales, in particular for complex cracks morphology. This versatile approach makes use of a regularized description of discontinuities through an additional phase field variable and strongly alleviates meshing problems by describing brittle cracking $[17,18]$. As compared with classical volume damage models, such regularized approach is directly connected to the theory of brittle crack propagation and removes mesh-sensitivity issues due to its natural nonlocal character. Furthermore, nucleation, interaction and arbitrary crack morphologies can thus be handled in regular meshes [19,20]. The method has proved to be highly suited to the simulation of microcracking in complex heterogeneous materials, e.g., see [20-22] and references therein. The phase field method may be approached both theoretically and practically, providing an elegant description for taking into account multi-physics effects on the failure phenomenon. The extension of this method to model thermo-mechanical problems is recently carried out and they can be found, for instance, in Refs. [23-26].

The present work is concerned with the development of a new multi-physics phase field model for simulating fracture problems for complex crack propagation induced by early-age shrinkage and heat of hydration in cementbased materials. In other words, our main goal is to present an effective multi-physics computational approach that combines the phase field model and a chemo-thermo-mechanical coupling scheme, and then apply it to model fracture in early-age concrete structures. The discretization of the problem is based on the finite element method (FEM). The new multi-physics model is essentially decoupled into three main problems: (i) chemo-thermal problem to determine temperature and hydration degree of the system; (ii) fracture problem to characterize cracking level; and (iii) mechanical problem to estimate the mechanical behavior of structure at a chosen time. The chemo-thermomechanical coupling scheme adopted here is mainly based on the ideas described in the previous works $[4,7,27]$. This scheme is highly suitable for representing the thermal/hydration evolution, or interpreting age effects and 
mechanical response. All those processes are incorporated into the variational phase field framework, and analysis of fracture phenomena of early-age concretes is then carried out and discussed. More importantly, the interaction among different processes is simulated in an efficient manner. The proposed model has applied to account for the most important multi-physics effects that occur in early-age concrete for the first few days after casting. Moreover, the influences of cracks on hydration and thermal problems are important, and they are hence addressed in this study. Compared with the conventional models available in the literature, the new approach presented here is able to represent all stages of fracture, such as crack nucleation, initiation, propagation, and also coalescence between difference cracks. One must notice that this is actually recognized as one difficult and challenging task for many present numerical methods such as smeared crack models [28,29], cohesive zone modeling [30-32], or extended finite element method [33]. In other words, the developed multi-physics computational approach goes beyond certain limitations of existing numerical models in modeling complex fracture induced by the heat of hydration, shrinkage strain in early-age concrete materials. Another important feature of the proposed model is its capability in dealing with the fracture at different scales thanks to non-local nature of phase field model. Specially, at micro/mesoscopic scales, in which cement-based materials exhibit strongly heterogeneous microstructures, the present method is capable of simulating extremely complex crack networks. In addition, the crack assessment requires here only two more parameters (i.e., fracture resistance and internal length), which can be easily identified by simple experimental test, see e.g., [34,35]. Hence the proposed model is strongly recommended to reproduce/validate experimental observations.

The rest of the paper is structured as follows. In Section 2, a brief description of fundamentals for the proposed method is derived. Then, mathematical model is introduced, in which we present a short review about the chemothermal model to evaluate the heat of hydration in Section 3.1 and its influences on the development of material properties in Section 3.2, respectively. The new phase field method for modeling complex crack propagation due to thermal and shrinkage strain is derived in Section 3.3. Section 4 details the numerical implementation of the present approach. In Section 5, the applicability, accuracy and performance of the developed approach are illustrated through practical numerical examples involving crack onset and propagation provided by hydration process. Some major conclusions drawn from this work are given in the conclusion section.

\section{Fundamentals}

Let $\Omega \subset \mathbb{R}^{D}$ be an open domain describing a hydration system at time $t$, containing a crack, as depicted in Fig. 1, with $D$ being the space dimension and $\partial \Omega$ its boundary. Besides two classical state variables, temperature $T(\mathbf{x})$ and displacement $\mathbf{u}(\mathbf{x})$, the state of system is defined by introducing two other state variables: the phase field variable $d(\mathbf{x})$ to represent damage level (i.e., $d=1$ for completely cracked area, and $d=0$ is intact material), and hydration degree $\alpha(\mathbf{x})$ to describe the level of hydration reaction between cement and water (i.e., $\alpha=1$ for full hydration).

A regularized framework is adopted to approximate the crack geometry by a smeared representation, in which the crack surface density function per unit volume of the solid, according to [17-19], is defined as $\gamma(d, \nabla d)=$ $\frac{1}{2 \ell} d^{2}+\frac{\ell}{2} \nabla d \cdot \nabla d$. It is important to notice that the definition of the crack surface density is based on the introduction of a regularization parameter, often called $\ell$, which controls the size of diffused zone and also affects the critical load of crack initiation [34,36,37].

The investigated system is subjected to the chemo-thermo-mechanical conditions, where $\bar{q}, \bar{T}, \bar{U}$, and $F$ are respectively prescribed thermal flux, prescribed temperature, prescribed displacement, and prescribed force. The radiation/convection between considered solid and environment with temperature $T_{a}$ is also taken into account. In this study, the small strain condition is assumed, and the proposed model incorporates four main problems: thermal, hydration, mechanical, and fracture processes. In this multi-physics context, the following important interactions among aforementioned fields should be taken into account, which are: (i) Interaction of temperature with hydration, mechanical and fracture processes; (ii) Interaction of hydration with mechanical, fracture and thermal processes; (iii) Interaction of mechanical with hydration, fracture and thermal processes; and (iv) Interaction of fracture with mechanical, hydration and thermal processes.

In this study, we are however trying to capture the effect of hydration reaction induced crack propagation by an appropriate simplest model. The influences of mechanical effects on thermal/hydration process (in point (iii) above) are hence neglected for the simplicity purpose. The other interactions are all included as depicted in Fig. 2, such as the interdependence of fracture process and thermal/mechanical/hydration processes, or between thermal process and 


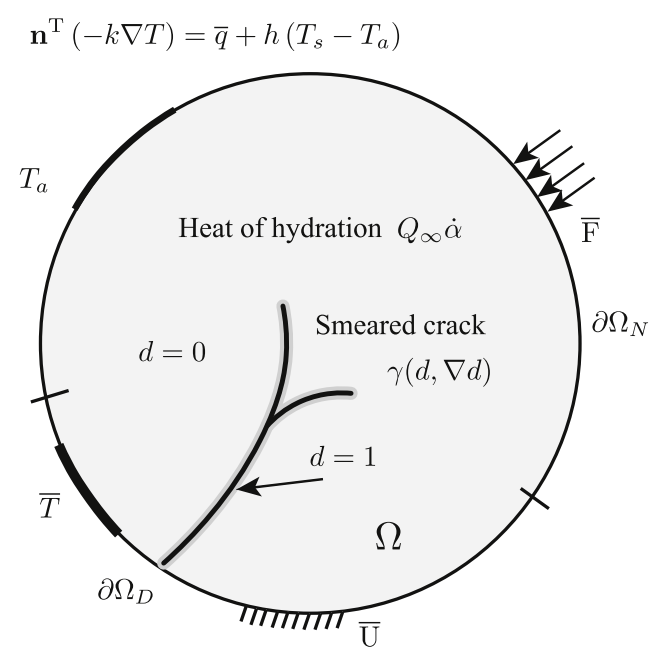

Fig. 1. Schematic representation of the investigated system: a solid body containing cracks, in which the hydration reaction takes place during the hardening process.

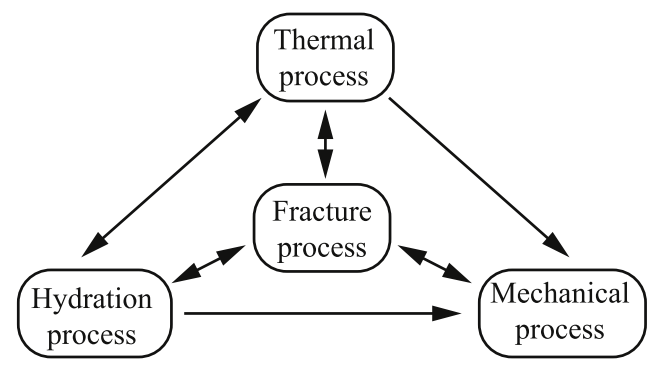

Fig. 2. Schematic representation of the interactions among different multi-physics processes.

hydration process. It is important to note that, for "bridging interactions", ${ }^{1}$ we can always capture the influences of mechanical problem on thermal/hydration problems. Moreover, the influences of creep strain and moisture diffusion are also not considered in this model. ${ }^{2}$

The new multi-physics model is decoupled into three main problems: (1) the chemo-thermal problem to determine the temperature and hydration degree of the system; (2) the fracture problem to characterize cracking level; and (3) the mechanical problem to estimate the mechanical behavior of the structure at a chosen time. Herein, the crack propagation is described by means of the phase field model, based on a regularized formulation of a sharp crack description as described above. One major advantage of the developed method is that it alleviates the shortcomings of re-meshing crack geometry by using a fixed mesh and a regularized description of the discontinuities. In addition, crack initiation can also be modeled in a straightforward manner. In contrast to volume damage models, usually implemented in non-linear codes, such a regularized approach is directly connected to the brittle failure theory of crack propagation.

\section{Mathematical model}

In this section, the mathematical descriptions of the coupled chemo-thermo-mechanical modeling in terms of the phase field model are presented.

\footnotetext{
1 An example to illustrate this property is given in the following, the mechanical process will directly affect the fracture, while the hydration/thermal process is directly influenced by fracture, i.e., the mechanics will affect thermal/hydration behavior via "bridging interactions"

2 The moisture diffusion often plays an important role in the thicker bodies. However, in the thinner systems, the characteristic time for this process is much slower than that one of the heating process. Hence the effects of moisture diffusion can be neglected, see e.g., [5] for more details.
} 


\subsection{Hydration heat}

The thermodynamic equilibrium of a concrete domain under thermal transient is governed by the Fourier's law, which includes the heat release due to hydration reaction

$$
\rho c \dot{T}=\nabla(\boldsymbol{k} \nabla T)+Q_{\infty} \dot{\alpha},
$$

where $\rho c$ is the volumetric heat capacity; the second term of the right hand, $Q_{\infty} \dot{\alpha}$, describes the heat release due to hydration reaction, in which $\dot{\alpha}$ is the hydration rate, and $Q_{\infty}$ represents the potential heat of the hydration reaction (the total heat that would be released upon full hydration of all cement $\alpha=1$ ); $\boldsymbol{k}$ is the local thermal conductivity matrix influenced by fracture process, and is determined as

$$
\boldsymbol{k}=(\xi(g(d)-1)+1) \boldsymbol{k}_{0}
$$

in which $\boldsymbol{k}_{0}$ is the initial thermal conductivity, $g(d)=(1-d)^{2}+\zeta$ is the quadratic degradation function, satisfying $g(0) \approx 1, g(1) \approx 0$ and $g^{\prime}(1)=0$ [19]. A small parameter $\zeta \ll 1$ is introduced to maintain the well-posedness of the system for partially broken parts of the domain. The parameter $\xi$ is used to describe two situations, $\xi=0$ for the case when the heat flux is not affected by a crack, and $\xi=1$ means that no heat will flow through cracks, or cracks are insulating [38]. The local thermal conductivity could be influenced by hydration process, as discussed in the previous works $[4,39]$. However, in the present investigation for the sake of simplicity $\boldsymbol{k}_{0}$ is considered to be independent from the hydration degree.

The associated boundary conditions are as follows

$$
T=\bar{T} \quad \text { on } \quad \partial \Omega_{D},
$$

and

$$
\mathbf{n}^{\mathrm{T}}(-\boldsymbol{k} \nabla T)=\bar{q}+h\left(T_{s}-T_{a}\right) \quad \text { on } \quad \partial \Omega_{N},
$$

where $\bar{T}$ and $\bar{q}$ are respectively the prescribed temperature, heat flux at the boundary $\Omega_{D}$ (Dirichlet part) and $\Omega_{N}$ (Neumann part). Second term on right hand of Eq. (4) represents the convection conditions, where $T_{s}, T_{a}$ are the body surface and air temperature, respectively; $h$ is the convection/radiation coefficient that could be chosen dependent upon a given wind speed at the solid surface and upon a given air temperature (see Appendix A).

The evolution of heat release due to the hydration process is assumed to be governed by the Arrhenius law, and by neglecting the effects of mechanical process [40], it is defined as follows:

$$
Q_{\infty} \dot{\alpha}=g(d) A_{T} f(\alpha) e^{\left(\frac{-E_{a}}{R T}\right)},
$$

or in other form

$$
\dot{\alpha}=g(d) \mathcal{A}_{\alpha} e^{\left(\frac{-E_{a}}{R T}\right)},
$$

in which $\mathcal{A}_{\alpha}=\frac{A_{T}}{Q_{\infty}} f(\alpha)$ is identified as the chemical affinity of the hydration reaction [40,41].

In Eq. (5), $E_{a}$ is the activation energy characterizing the rate of heat generation, $R=8.314 \times 10^{-3}\left[\mathrm{~kJ} \mathrm{~K}^{-1} \mathrm{~mol}^{-} 1\right]$ is the ideal gas constant. The parameter $A_{T}$ stands for the ratio of the maximum value of the heat production rate to the latent hydration heat for a normalized definition of the hydration function. The degradation function $g(d)$ is added to ensure that no heat of hydration produced at the cracked area takes place. The chemical affinity function $f(\alpha)$, represents the evolution of the normalized heat production rate in terms of the hydration degree. In general, there are many possible choices for this particular function. It could be, for instance, either a piecewise linear or exponential approximation of the experimental data (calorimetric characterization of the cement hydration) [7,10,41]. In the present contribution, the power form of the evolution function, $f(\alpha)$, for the normalized heat production rate is adopted as presented in [27]

$$
f(\alpha)=\left(\frac{\alpha}{a}\right)^{b}\left(\frac{1-\alpha}{1-a}\right)^{c} .
$$


Basically, three constants $a, b$, and $c$ given in Eq. (7) are identified based on experimental data, wherein $a$ defines the hydration degree for the maximum value of the normalized cement hydration function, as determined from experimental calorimetric tests, the exponent $b<1$ is chosen to obtain well-posed problem. The stationary condition yields the relation $a c=(1-a) b$, meaning that one of the exponents is used as a fitting parameter, see e.g., [10,27,41] for more details.

Consequently, by combining Eqs. (1) and (5), a chemo-thermal process is described, which is based on a set of coupled equations to be solved in the domain $\Omega$

$$
\begin{cases}\rho c \dot{T}=\nabla(\boldsymbol{k} \nabla T)+Q_{\infty} \dot{\alpha} & \text { in } \Omega, \\ Q_{\infty} \dot{\alpha}=g(d) A_{T} f(\alpha) \exp \left(E_{a} / R T\right) & \text { in } \Omega, \\ T=\bar{T} & \text { on } \partial \Omega_{D}, \\ \mathbf{n}^{\mathrm{T}}(-\boldsymbol{k} \nabla T)=\bar{q}+h\left(T_{s}-T_{a}\right) & \text { on } \partial \Omega_{N} .\end{cases}
$$

\subsection{Age effects}

The chemical processes that occur in the hardening cement paste strongly affect its mechanical properties, the Young's modulus $E$, the Poisson's ratio $v$, and the fracture resistance $g_{c}$. The development of material strength due to the age effects can be defined as a function of hydration degree according to the idea proposed in $[7,42,43]$

$$
\left\{\begin{array}{l}
E(\alpha)=E_{\infty} \bar{\alpha}^{\alpha_{E}} \\
v(\alpha)=0.18 \sin \frac{\pi \alpha}{2}+0.5 e^{-10 \alpha} \\
g_{c}(\alpha)=g_{c \infty} \bar{\alpha}^{\alpha_{g c}}
\end{array}\right.
$$

where $E_{\infty}$ and $g_{c \infty}$ are respectively the final Young's modulus and final fracture energy; $\alpha_{E}$ and $\alpha_{g_{c}}$ are two threshold constants, which define the moment when material starts having its strength. According to [4,43], one possible choice for $\bar{\alpha}^{\alpha}, \bar{\alpha}^{\alpha_{c c}}$ is

$$
\bar{\alpha}^{\alpha_{E}}=\left\langle\frac{\alpha-\alpha_{E}}{1-\alpha_{E}}\right\rangle_{+} \quad \text { and } \quad \bar{\alpha}^{\alpha_{g_{c}}}=\left\langle\frac{\alpha-\alpha_{g_{c}}}{1-\alpha_{g_{c}}}\right\rangle_{+},
$$

where $\langle.\rangle_{+}$denotes the positive operator.

\subsection{Chemo-thermo-mechanical coupling phase field model}

In this subsection, we present basic concepts as well as the main ideas of the phase field model for fracture. For more details about the practical implementation aspects, we recommend interested readers to refer to e.g. Refs. [19,20]. The free energy is assumed to depend on the primary variables $\{\mathbf{u}, \alpha, T, d, \nabla d\}$, which can split into an elastic part $E_{e}$ of the elastic stored energy, fracture part $E_{d}$ of fracture surface energy, and thermo-chemical part $E_{T \alpha}$

$$
E(\mathbf{u}, \alpha, T, d, \nabla d)=E_{e}(\mathbf{u}, d, \alpha, T)+E_{d}(d, \nabla d)+E_{T \alpha}(T, \alpha)
$$

By assuming the small strains condition, the regularized form of the energy describing the cracked structure induced by early-age shrinkage and hydration heat can be expressed as follows

$$
E(\mathbf{u}, \alpha, T, d, \nabla d)=\int_{\Omega} \psi^{e}\left(\varepsilon^{e}, d\right) \mathrm{d} \Omega+\int_{\Omega} \psi^{d}(d, \nabla d) \mathrm{d} \Omega+\int_{\Omega} \psi^{T \alpha}(T, \alpha) \mathrm{d} \Omega .
$$

The first term $\psi^{e}$ is the density of the elastic energy, defined as a function of the phase field $d$ and the elastic strain $\boldsymbol{\varepsilon}^{e}$, which is assumed to be affected by thermal expansion strain $\boldsymbol{\varepsilon}^{\text {th }}$ and autogenous shrinkage strain $\boldsymbol{\varepsilon}^{\text {au }}$, yielding

$$
\boldsymbol{\varepsilon}^{e}=\frac{1}{2}\left((\nabla \mathbf{u})^{\mathrm{T}}+(\nabla \mathbf{u})\right)-\boldsymbol{\varepsilon}^{\mathrm{th}}-\varepsilon^{\mathrm{au}}
$$

with

$$
\boldsymbol{\varepsilon}^{\text {th }}=\beta\left(T-T_{0}\right) \mathbf{I} \quad \text { and } \quad \boldsymbol{\varepsilon}^{\mathrm{au}}=-\kappa\left\langle\frac{\alpha-\alpha_{a u}}{1-\alpha_{a u}}\right\rangle_{+} \mathbf{I},
$$

in which $\beta$ is the thermal expansion coefficient, $\mathbf{I}$ is the identity tensor, and a material constant $\kappa$ represents the evolution of autogenous shrinkage when hydration degree is greater than the mechanical percolation threshold $\alpha_{a u}$. 
The density of fracture energy $\psi^{d}$ in Eq. (12), describes the amount of energy released upon the creation of new fracture surface, which is expressed by

$$
\psi^{d}=g_{c}(\alpha) \gamma(d, \nabla d)
$$

where $g_{c}(\alpha)$ defines the fracture resistance influenced by hydration.

The last term in (12) is the thermo-chemical contributions to the stored energy per unit volume $\psi^{T \alpha}(T, \alpha)$, which is chosen following [41], reads as

$$
\psi^{T \alpha}=-\frac{\rho c}{2 T_{0}}\left(T-T_{0}\right)^{2}+\frac{Q_{\infty} \alpha}{T_{0}}\left(T-T_{0}\right)+h(\alpha)
$$

where $h(\alpha)$ describes the purely chemical contribution. An explicit formulation for $h(\alpha)$ can be found e.g. in [40,41].

The total energy is then rewritten as $E=\int_{\Omega} \psi \mathrm{d} \Omega$, in which $\psi$ is the total density energy defined as follows

$$
\psi=\psi^{e}+\psi^{d}+\psi^{T \alpha} .
$$

Based on Ref. [19], the unilateral contact formulation is thus chosen to ensure damage induced by traction only, (assuming isotropic elastic behavior of the body) through

$$
\psi^{e}=g(d) \psi^{e+}\left(\varepsilon^{e}\right)+\psi^{e-}\left(\varepsilon^{e}\right)
$$

with

$$
\psi^{e \pm}\left(\varepsilon^{e}\right)=\frac{\lambda(\alpha)}{2}\left[\left\langle\operatorname{tr} \varepsilon^{e}\right\rangle_{ \pm}\right]^{2}+\mu(\alpha) \operatorname{tr}\left[\left(\varepsilon^{e \pm}\right)^{2}\right]
$$

where $\varepsilon^{e+}$ and $\varepsilon^{e-}$ are, respectively, the extensive and compressive modes of the elastic strain field $\boldsymbol{\varepsilon}^{e}=\boldsymbol{\varepsilon}^{e+}+\boldsymbol{\varepsilon}^{e-}$.

Applying the principle of maximum dissipation to Eq. (12) yields the set of coupled equations to be solved on the domain $\Omega \in \mathbb{R}^{D}$ associated with the structure, with boundary $\partial \Omega$ and outward normal $\mathbf{n}$, to determine $d(\mathbf{x}, t)$ at time $t$ (see Appendix B and Refs, e.g., [20,44,45] for more details):

$$
\begin{cases}2(1-d) \mathcal{H}-\left(d-\ell^{2} \Delta d\right)=0 & \text { in } \Omega, \\ d(\mathbf{x})=1 & \text { on } \Gamma, \\ \nabla d(\mathbf{x}) \cdot \mathbf{n}=0 & \text { on } \Omega,\end{cases}
$$

where $\Gamma$ is the preexisting crack curve of dimension $D-1$ within $\Omega$. The local crack driving force $\mathcal{H}(\mathbf{x}, t)$ is introduced to ensure the positive of Eq. (B.15). It also describes a dependence on history [19], and to make loading-unloading possible

$$
\mathcal{H}(\mathbf{x}, t)=\max _{\tau \in[0, t]}\left\{\frac{\ell}{g_{c}(\alpha)} \psi^{e+}(\mathbf{x}, \tau)\right\} .
$$

The local history field $\mathcal{H}(\mathbf{x}, t)$ contains the maximum reference energy, or a measure for the maximum tensile strain obtained in the deformation history, which provides a very transparent representation of the balance equation that governs the diffusive crack topology in Eq. (20). For more detailed information, curious readers should refer to references, e.g., [19,20], in which the loading-unloading character of the proposed approach is also demonstrated by an experiment of cyclic loading.

The irreversible character of the crack evolution law described in Eq. (20) can be checked by following. For a homogeneous damage problem with $\nabla d=0$, the relation between $d$ and $\mathcal{H}$ is

$$
d=\frac{2 \mathcal{H}}{1+2 \mathcal{H}} \in[0,1]
$$

and

$$
\dot{d}=\frac{2 \dot{\mathcal{H}}}{(1+2 \mathcal{H})^{2}} \geq 0
$$

is ensured with the definition of $\mathcal{H}$ in Eq. (21). Note that, the irreversibility of the crack for the non-homogeneous damage problem in (20) can be also verified in the same way by using the maximum principle of elliptic partial differential equations proposed in [46]. 
The Euler-Lagrange equations of the variational principle for energy minimization to Eq. (12) are constructed as follows:

$$
\mathbf{u}(\mathbf{x})=\operatorname{Arg}\left\{\inf _{\mathbf{u} \in \mathcal{S}_{u}}\left(E(\mathbf{u}, \alpha, T, d, \nabla d)-W^{e x t}\right)\right\}
$$

where $\mathcal{S}_{u}=\left\{\mathbf{u} \mid \mathbf{u}(\mathbf{x})=\overline{\mathbf{u}}\right.$ on $\left.\partial \Omega_{D}, \quad \mathbf{u} \in H^{1}(\Omega)\right\}$ and $W^{\text {ext }}=\int_{\Omega} \mathbf{f} \cdot \mathbf{u} \mathrm{d} \Omega+\int_{\partial \Omega_{N}} \overline{\mathbf{F}} \cdot \mathbf{u} \mathrm{d} \Gamma$ with $\mathbf{f}$ and $\overline{\mathbf{F}}$ being body forces and prescribed traction over the boundary $\partial \Omega_{N}$, yields the mechanical problem defined by

$$
\begin{cases}\nabla \cdot \boldsymbol{\sigma}+\mathbf{f}=\mathbf{0} & \text { in } \Omega, \\ \mathbf{u}=\overline{\mathbf{u}} & \text { on } \partial \Omega_{D}, \\ \boldsymbol{\sigma} \mathbf{n}=\overline{\mathbf{F}} & \text { on } \partial \Omega_{N} .\end{cases}
$$

In Eq. (25), $\sigma$ is the second-order Cauchy stress tensor, $\overline{\mathbf{u}}$ are the prescribed displacement on the boundary $\partial \Omega_{D}$. The constitutive law described in Eq. (B.5) is thus expressed as

$$
\boldsymbol{\sigma}=g(d)\left(\lambda(\alpha)\left\langle\operatorname{tr} \boldsymbol{\varepsilon}^{e}\right\rangle_{+} \mathbf{1}+2 \mu(\alpha) \boldsymbol{\varepsilon}^{e+}\right)+\left(\lambda(\alpha)\left\langle\operatorname{tr} \boldsymbol{\varepsilon}^{e}\right\rangle_{-} \mathbf{1}+2 \mu(\alpha) \boldsymbol{\varepsilon}^{\boldsymbol{e}^{-}}\right) .
$$

\section{Discretization and numerical implementation}

The discretization of the system of governing equations at element level using the FEM for all field variables can be expressed as follows:

$$
\begin{aligned}
& T=\mathbf{N}_{T} \mathbf{T}^{e}, \quad \alpha=\mathbf{N}_{\alpha} \boldsymbol{\alpha}^{e}, \quad \mathbf{u}=\mathbf{N}_{u} \mathbf{u}^{e}, \quad d=\mathbf{N}_{d} \mathbf{d}^{e}, \\
& \nabla T=\mathbf{B}_{T} \mathbf{T}^{e}, \quad \nabla \mathbf{v}^{s}=\mathbf{B}_{u} \mathbf{u}^{e}, \quad \nabla d=\mathbf{B}_{d} \mathbf{d}^{e}, \\
& \tilde{T}=\mathbf{N}_{T} \tilde{\mathbf{T}}^{e}, \quad \tilde{\alpha}=\mathbf{N}_{\alpha} \tilde{\boldsymbol{\alpha}}^{e},
\end{aligned}
$$

where $\mathbf{T}^{e}, \tilde{\mathbf{T}}^{e}, \boldsymbol{\alpha}^{e}, \tilde{\boldsymbol{\alpha}}^{e}, \mathbf{u}^{e}$, and $\mathbf{d}^{e}$ are, respectively, nodal temperature components, nodal incremental temperature components, nodal hydration degree components, nodal incremental hydration components, nodal displacement components, and nodal phase field components. $\mathbf{N}_{T, \alpha, u, d}$ and $\mathbf{B}_{T, \alpha, u, d}$ are vectors/matrices of shape functions as well as matrices of shape functions derivatives for scalar/tensor fields.

\subsection{Chemo-thermal problem} form

Using the Galerkin method, we can rewrite the basic coupled chemo-thermal problem, Eq. (8), in the following

$$
\left\{\begin{array}{l}
\int_{\Omega} \mathbf{B}_{T}^{\mathrm{T}} \boldsymbol{k} \mathbf{B}_{T} \mathrm{~d} \Omega \mathbf{T}^{e}+\int_{\Omega} \mathbf{N}_{T}^{\mathrm{T}} \rho c \mathbf{N}_{T} \mathrm{~d} \Omega \dot{\mathbf{T}}^{e}-\int_{\Omega} \mathbf{N}_{\alpha}^{\mathrm{T}} Q_{\infty} \mathbf{N}_{\alpha} \mathrm{d} \Omega \dot{\boldsymbol{\alpha}}^{e}+\int_{\partial \Omega} \mathbf{N}_{T}^{\mathrm{T}}\left(\mathbf{n}^{T} \mathbf{q}\right) \mathrm{d} S=0, \\
\int_{\Omega} \mathbf{N}_{\alpha}^{\mathrm{T}} Q_{\infty} \mathbf{N}_{\alpha} \mathrm{d} \Omega \dot{\boldsymbol{\alpha}}^{e}-\int_{\Omega} \mathbf{N}_{\alpha}^{\mathrm{T}}\left(g(d) A_{T} f(\alpha) \exp \left(-E_{a} / R T\right)\right) \mathrm{d} \Omega=0 \\
\int_{\partial \Omega} \mathbf{N}_{T}^{\mathrm{T}}\left(\mathbf{n}^{T} \mathbf{q}\right) \mathrm{d} S=\int_{\partial \Omega_{q}} \mathbf{N}_{T}^{\mathrm{T}} \bar{q} \mathrm{~d} S+\int_{\partial \Omega_{c}} \mathbf{N}_{T}^{\mathrm{T}} h \mathbf{N}_{T} \mathrm{~d} S \mathbf{T}^{e}-\int_{\partial \Omega_{c}} \mathbf{N}_{T}^{\mathrm{T}} h T_{a} \mathrm{~d} S,
\end{array}\right.
$$

and in the matrix form as detailed in Appendix C)

$$
\left\{\begin{array}{l}
\mathbf{K}_{T} \mathbf{T}^{e}+\mathbf{H}_{T} \dot{\mathbf{T}}^{e}+\mathbf{Q}_{c} \mathbf{T}^{e}+\mathbf{F}_{q}-\mathbf{F}_{c}-\mathbf{F}_{\alpha}=0 \\
\mathbf{Q}_{\alpha} \dot{\boldsymbol{\alpha}}^{e}-\mathbf{F}_{\alpha}=0
\end{array}\right.
$$

A standard Newton-Raphson method is used to solve the non-linear problem Eq. (29). Introducing the residual $\mathcal{R}_{T}=\mathbf{K}_{T} \mathbf{T}^{e}+\mathbf{H}_{T} \dot{\mathbf{T}}^{e}+\mathbf{Q}_{c} \mathbf{T}^{e}+\mathbf{F}_{q}-\mathbf{F}_{c}-\mathbf{F}_{\alpha}$ and $\mathcal{R}_{\alpha}=\mathbf{Q}_{\alpha} \dot{\boldsymbol{\alpha}}^{e}-\mathbf{F}_{\alpha}$ yields the following linear system that is solved at each iteration

$$
\left\{\begin{array}{c}
\frac{\partial \mathcal{R}_{T}}{\partial \mathbf{T}^{e}} \tilde{\mathbf{T}}=-\mathcal{R}_{T}, \\
\frac{\partial \boldsymbol{\mathcal { R }}_{\alpha}}{\partial \boldsymbol{\alpha}^{e}} \tilde{\boldsymbol{\alpha}}=-\boldsymbol{\mathcal { R }}_{\alpha} .
\end{array}\right.
$$

Using the Euler method for time integration $\dot{x}_{n+1}=\left(x_{n+1}-x_{n}\right) / \Delta t$, the tangential matrix at time step $n+1$ reads

$$
\frac{\partial \boldsymbol{\mathcal { R }}_{T}}{\partial \mathbf{T}_{n+1}^{e}}=\mathbf{K}_{T}+\frac{1}{\Delta t} \mathbf{H}_{T}+\mathbf{Q}_{c}-\frac{\partial \mathbf{F}_{\alpha}}{\partial \mathbf{T}_{n+1}^{e}},
$$


and

$$
\frac{\partial \boldsymbol{R}_{\alpha}}{\partial \boldsymbol{\alpha}_{n+1}^{e}}=\frac{1}{\Delta t} \mathbf{Q}_{\alpha}-\frac{\partial \mathbf{F}_{\alpha}}{\partial \boldsymbol{\alpha}_{n+1}^{e}},
$$

in which

$$
\begin{aligned}
\frac{\partial \mathbf{F}_{\alpha}}{\partial \mathbf{T}_{n+1}^{e}} & =\int_{\Omega} \mathbf{N}_{T}^{\mathrm{T}}\left(g(d) A_{T} f(\alpha) \frac{E_{a}}{R T^{2}} \exp \left(-E_{a} / R T\right)\right) \mathbf{N}_{T} \mathrm{~d} \Omega, \\
\frac{\partial \mathbf{F}_{\alpha}}{\partial \boldsymbol{\alpha}_{n+1}^{e}} & =\int_{\Omega} \mathbf{N}_{\alpha}^{\mathrm{T}}\left(g(d) A_{T} \frac{\partial f(\alpha)}{\alpha} \exp \left(-E_{a} / R T\right)\right) \mathbf{N}_{\alpha} \mathrm{d} \Omega,
\end{aligned}
$$

\subsection{Mechanical problem}

The unilateral contact formulation described in Eq. (19) is based on the spectral decomposition of the elastic strain tensor $\boldsymbol{\varepsilon}^{e}$. Let $\left\{\varepsilon^{e}\right\}_{i, i=1 \ldots \delta}$ and $\{\boldsymbol{n}\}_{i, i=1 \ldots \delta}$ be the principal strains and the principal strain directions, respectively. The positive and negative parts of the elastic strain can thus be expressed as [19]

$$
\boldsymbol{\varepsilon}^{e \pm}=\sum_{i=1}^{\delta}\left\langle\varepsilon_{i}^{e}\right\rangle_{ \pm} \boldsymbol{n}_{i} \otimes \boldsymbol{n}_{i}
$$

and its derivatives with respect to the elastic strain defines two projection tensor are

$$
\mathbb{P}^{ \pm}\left(\varepsilon^{e}\right)=\partial_{\varepsilon^{e}}\left[\varepsilon^{e \pm}\left(\varepsilon^{e}\right)\right],
$$

which are isotropic tensors, nonlinear functions of $\boldsymbol{\varepsilon}^{e}$, providing the nonlinearity in the mechanical problem.

To avoid this nonlinearity related to the aforementioned spectral decomposition, we adopted the shifted strain tensor split algorithms as previously proposed by the present authors in Ref. [20]. Within the context of incremental scheme, the projection tensors at the investigated time step $n+1$ will be evaluated based on the results from previous time step $n$, by the following expression ${ }^{3}$ :

$$
\boldsymbol{\varepsilon}_{n+1}^{e \pm}\left(\boldsymbol{\varepsilon}_{n}^{e}\right) \simeq \mathbb{P}^{ \pm}\left(\boldsymbol{\varepsilon}_{n}^{e}\right): \boldsymbol{\varepsilon}_{n+1}^{e}, \quad \text { and }\left\langle\operatorname{tr} \boldsymbol{\varepsilon}_{n+1}^{e}\right\rangle_{ \pm} \simeq \mathcal{R}^{ \pm}\left(\boldsymbol{\varepsilon}_{n}^{e}\right) \operatorname{tr} \boldsymbol{\varepsilon}_{n+1}^{e}
$$

Setting $\mathcal{R}^{ \pm}\left(\boldsymbol{\varepsilon}_{n}^{e}\right) \equiv \mathcal{R}^{ \pm}$and $\mathbf{P}^{ \pm}\left(\boldsymbol{\varepsilon}_{n}^{e}\right) \equiv \mathbf{P}^{ \pm}$, where $\mathbf{P}^{ \pm}\left(\varepsilon_{n}^{e}\right)$ are the matrix forms associated with the fourth-order tensors $\mathbb{P}^{ \pm}$, then the stress can be expressed at time $t_{n+1}$ as

$$
[\boldsymbol{\sigma}]=\left[g(d)\left(\lambda(\alpha) \mathcal{R}^{+}[\mathbf{1}] \cdot[\mathbf{1}]+2 \mu(\alpha) \mathbf{P}^{+}\right)+\left(\lambda(\alpha) \mathcal{R}^{-}[\mathbf{1}] \cdot[\mathbf{1}]+2 \mu(\alpha) \mathbf{P}^{-}\right)\right]\left[\boldsymbol{\varepsilon}^{e}\right]
$$

The matrix form of elastic tensor in Voigt notation can be written as follows:

$$
\mathbf{C}(d, \alpha)=\left[g(d)\left(\lambda(\alpha) \mathcal{R}^{+}[\mathbf{1}] \cdot[\mathbf{1}]+2 \mu(\alpha) \mathbf{P}^{+}\right)+\left(\lambda(\alpha) \mathcal{R}^{-}[\mathbf{1}] \cdot[\mathbf{1}]+2 \mu(\alpha) \mathbf{P}^{-}\right)\right]
$$

From Eqs. (25), (38), the linear system of equations is obtained for time step $n+1$ :

$$
\mathbf{K}_{u} \mathbf{u}_{n+1}=\mathbf{F}_{u},
$$

where

$$
\mathbf{K}_{u}=\int_{\Omega} \mathbf{B}_{u}^{\mathrm{T}} \mathbf{C}(d, \alpha) \mathbf{B}_{u} \mathrm{~d} \Omega \quad \text { and } \quad \mathbf{F}_{u}=\int_{\Omega} \mathbf{B}_{u}^{\mathrm{T}}\left(\left[\boldsymbol{\varepsilon}^{\mathrm{th}}\right]-\left[\boldsymbol{\varepsilon}^{\mathrm{au}}\right]\right)[\mathbf{1}] \mathrm{d} \Omega
$$

\subsection{Phase field problem}

Using the variational principle for Eq. (20), and based on the above discretization, the finite element equation is given by

$$
\mathbf{K}_{d} \mathbf{d}_{n+1}=\mathbf{F}_{d},
$$

\footnotetext{
3 The computation of $\mathbb{P}^{ \pm}\left(\varepsilon^{e}\right)$ can be expressed thanks to the algorithm presented in Miehe and Lambrecht [47].
} 
where

$$
\begin{aligned}
\mathbf{K}_{d} & =\int_{\Omega} \mathbf{N}_{d}^{\mathrm{T}}\left(2 \mathcal{H}_{n}+1\right) \mathbf{N}_{d} \mathrm{~d} \Omega+\int_{\Omega} \ell^{2} \mathbf{B}_{d}^{\mathrm{T}} \mathbf{B}_{d} \mathrm{~d} \Omega \\
\mathbf{F}_{d} & =\int_{\Omega} 2 \mathbf{N}_{d}^{\mathrm{T}} \mathcal{H}_{n} \mathrm{~d} \Omega
\end{aligned}
$$

\subsection{Key steps of solution algorithm}

Here the main steps of the solution procedure or pseudo codes are briefly presented:

\section{- Initialization}

(i) Initialize the temperature field $T_{0}(\mathbf{x})$, the hydration degree field $\alpha_{0}(\mathbf{x})$ and its time derivatives $\dot{T}_{0}(\mathbf{x}), \dot{\alpha}_{0}(\mathbf{x})$

(ii) Initialize the displacement field $\mathbf{u}_{0}(\mathbf{x})$, the phase field $d_{0}(\mathbf{x})$, and the strain-history functional $\mathcal{H}_{0}=0$.

- FOR $t^{n+1} \leq t^{\max }$, given $T_{n}, \dot{T}_{n}, \alpha_{n}, \dot{\alpha}_{n} \mathbf{u}_{n}, d_{n}$ and $\mathcal{H}_{n}$.

\section{(1) Chemo-thermal problem}

Set $T_{k}=T_{n}, \dot{T}_{k}=\dot{T}_{n}, \alpha_{k}=\alpha_{n}$, and $\dot{\alpha}_{k}=\dot{\alpha}_{n}$

WHILE Error $>10^{-6}$

. (i) Compute the tangential matrix $\left(\frac{\partial \boldsymbol{\mathcal { R }}_{T}}{\partial \mathbf{T}_{n+1}^{e}}\right)_{T_{k}},\left(\frac{\partial \boldsymbol{\mathcal { R }}_{\alpha}}{\partial \boldsymbol{\alpha}_{n+1}^{e}}\right)_{\alpha_{k}}$ and residual $\left(\boldsymbol{\mathcal { R }}_{T}\right)_{T_{k}},\left(\boldsymbol{\mathcal { R }}_{\alpha}\right)_{\alpha_{k}}{ }^{4}$ by following Eqs. (29), (31), (32).

. (ii) Compute $\tilde{\mathbf{T}}_{k+1}$ and $\tilde{\boldsymbol{\alpha}}_{k+1}$ following Eq. (30)

. (iii) Update $\boldsymbol{T}_{k+1}=\boldsymbol{T}_{k}+\tilde{\boldsymbol{T}}_{k+1}$ and $\boldsymbol{\alpha}_{k+1}=\boldsymbol{\alpha}_{k}+\tilde{\boldsymbol{\alpha}}_{k+1}$

. (iv) Compute Error $=\max \left(\left\|\tilde{\boldsymbol{T}}_{k+1}\right\|,\left\|\tilde{\boldsymbol{\alpha}}_{k+1}\right\|\right)$

\section{END}

Update $T_{n+1}=T_{k+1}, \dot{T}_{n+1}=\dot{T}_{k+1}, \alpha_{n+1}=\alpha_{k+1}$, and $\dot{\alpha}_{n+1}=\dot{\alpha}_{k+1}$

(2) Account for age effects

Material parameters are updated based on Eq. (9) by taking into account the age effects

(3) Phase field problem

(i) Compute history field $\mathcal{H}_{n}(\mathbf{x}, \tau)$ by Eq. (21)

. (ii) Compute phase field $d_{n+1}(\mathbf{x})$ by solving problem Eq. (42)

\section{(4) Displacement problem}

. (i) Compute $\mathbb{P}_{n}^{ \pm}, \mathcal{R}_{n}$, following $[19,20]$

. (ii) Compute displacement field $\mathbf{u}_{n+1}(\mathbf{x})$ by solving problem Eq. (40)

$(.)_{n} \leftarrow(.)_{n+1}$ and go to (1).

\section{- END}

Remark: In this paper, the staggered algorithm is selected for updating both displacements and phase field because of its robustness. The energy functional defined in Eq. (12) is convex with respect to each of its arguments $u$ and $d$ separately, which ensures the existence of solution for the variational principle of energy minimization. In particular, the one-pass solution scheme as presented in, e.g., [19,26,37] is adopted for the present model. This scheme is extremely robust, requiring only one pass of the updating stage for a load increment. That is different from ones used staggered iterations (multi-pass solution) reported in [17,36,48], where additional Newton-Raphson iterations can be performed within a time step. Many contributions available in the literature, e.g., see [19,20,26,49,50], have shown existence and convergence results for this time discretization scheme. Note that, the strong iteration between fracture and mechanical problems can be accurately captured by such

\footnotetext{
4 The notation $(.)_{T_{k}, \alpha_{k}}$ means computing on the results of previous iteration $k$.
} 


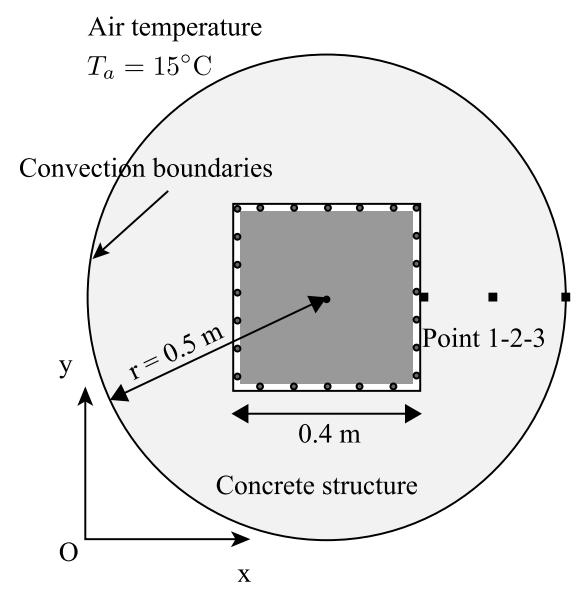

Fig. 3. Geometry and boundary conditions of considered structure, a circular surface containing a square hole is subjected to convection conditions.

staggered scheme, e.g., the interdependence of stress relaxation and energy dissipation. However, the solution seems to be affected by the time step. Therefore, a small time step or a small incremental loading is often needed to ensure the accuracy of numerical results, which has previously been shown in [20,51,52]. In this particular study, it is hence set by $\Delta t \leq 600 \mathrm{~s}$. Furthermore, it is worth mentioning that the fracture phenomena induced by hydration process are often slow. Therefore, the one-pass staggered scheme is used for the present problem as it could offer an acceptable solution.

\section{Numerical experiments and discussions}

In this section, numerical experiments will be provided to show the performance of the proposed approach. In particular, we study two numerical examples, one is for a homogeneous structure and the other is devoted to a strongly heterogeneous structure. In this paper, we additionally provide validation of the proposed model for its accuracy by comparing the estimated results with respect to appropriate reference solutions, as detailed in Appendix D.

\subsection{Cement ring structure: hydration reaction induced fracture analysis}

For the homogeneous structural analysis, we consider a circular solid made of concrete containing a square hole as schematically sketched in Fig. 3. A radius of $\mathrm{r}=0.5 \mathrm{~m}$ is selected, and the size of the square hole is $0.4 \mathrm{~m}$. The material parameters are taken following Ref. [7], and listed in Table 1. The critical hydration degree, in which cement material begins having its strength is chosen as follows: $\alpha_{E}=0.35$ for the Young's modulus and $\alpha_{g_{c}}=0.3$ for fracture resistance. The autogenous shrinkage strain is activated when the hydration greater than $\alpha_{a u}=0.115$ (see Eq. (14)). Concerning the internal length $\ell$ of the phase field model, as discussed in [34], by using an analysis of the traction of a one-dimensional bar, a rough relationship among $\ell$ and material parameters $\left(E, g_{c}\right)$ and the tensile strength $\sigma_{c}$ was constructed, and that reaches as

$$
\ell=\frac{27 E g_{c}}{256 \sigma_{c}^{2}}
$$

leading to $\ell \approx 12.5 \mathrm{~mm}$ with a tensile strength of concrete $\sigma_{c}=2.75 \mathrm{MPa}$.

Here, the crack is assumed to be conductive, i.e $\xi=0$ (see Eq. (2)). The initial conditions $T_{0}=20{ }^{\circ} \mathrm{C}$ and $\alpha_{0}=0.01$ are chosen for whole system. The structure is subjected to the convection boundary conditions, in which the radiation/convection coefficient is assumed to be independent of wind speed and $h=8\left[\mathrm{~W} /\left(\mathrm{m}^{2} \mathrm{~K}\right)\right]$. The ambient temperature is considered to be constant during the whole simulation, with $T_{a}=15^{\circ} \mathrm{C}$. Moreover, the $x$-displacements in the left-end and right-end of square hole are fixed, while $y$-displacements are free; on the lower-end and upper-end, $y$-displacements are fixed, while $x$-displacements are free. The structure is meshed using triangular elements with $h_{e}^{\max }=0.005 \mathrm{~m}$ and $h_{e}^{\min }=0.0025 \mathrm{~mm}$, satisfying the condition $\ell>2 h_{e}$ for having several elements inside the 
Table 1

Material properties (Ref. [7])

\begin{tabular}{llllll}
\hline Parameter & Value & Unit & Parameter & Value & Unit \\
\hline$\rho c$ & 2400 & $\mathrm{~kJ} /\left(\mathrm{K} \mathrm{m}^{3}\right)$ & $\alpha_{a u}$ & 0.115 & - \\
$k$ & 2.8 & $\mathrm{~W} /(\mathrm{m} \mathrm{K})$ & $\alpha_{E}$ & 0.35 & - \\
$Q_{\infty}$ & 117840 & $\mathrm{~kJ} / \mathrm{m}^{3}$ & $\alpha_{g_{c}}$ & 0.30 & - \\
$h$ & 8 & $\mathrm{~W} /\left(\mathrm{m}^{2} \mathrm{~K}\right)$ & $\ell$ & 0.0125 & $\mathrm{~m}$ \\
$\beta$ & 15 & $\mu \mathrm{m} /(\mathrm{m} \mathrm{K})$ & $v$ & 0.22 & - \\
$\kappa$ & 75 & $\mu \mathrm{m} / \mathrm{m}$ & $T_{a}$ & 15 & ${ }^{\circ} \mathrm{C}$ \\
$E_{\infty}$ & 35 & $\mathrm{GPa}$ & $A_{T}$ & 0.215 & $\mathrm{GW} / \mathrm{kg}$ \\
$g_{c}$ & 25 & $\mathrm{~N} / \mathrm{m}$ & $E_{a}$ & 44.929 & $\mathrm{~kJ} / \mathrm{mol}$ \\
\hline
\end{tabular}
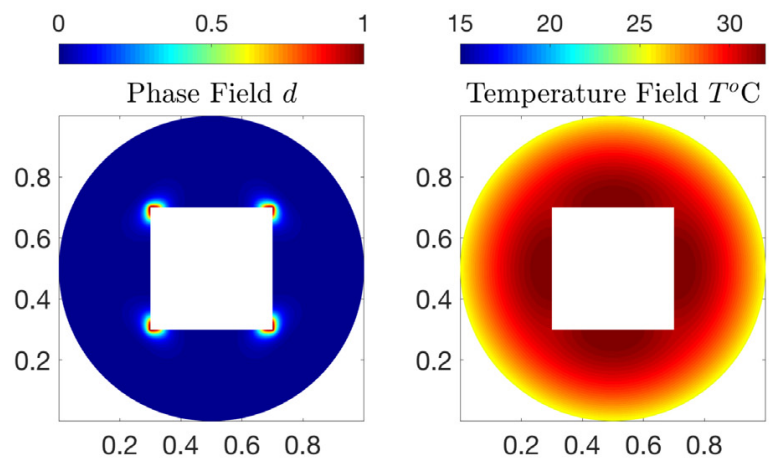

(a) time $t=25$ hours

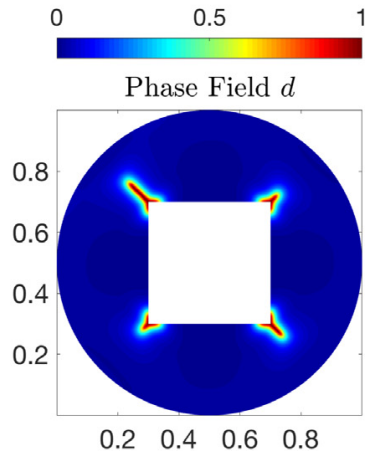

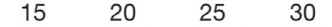

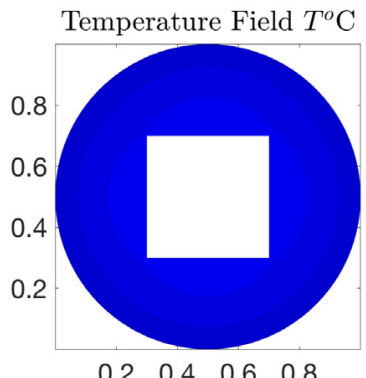

(b) time $t=112$ hours
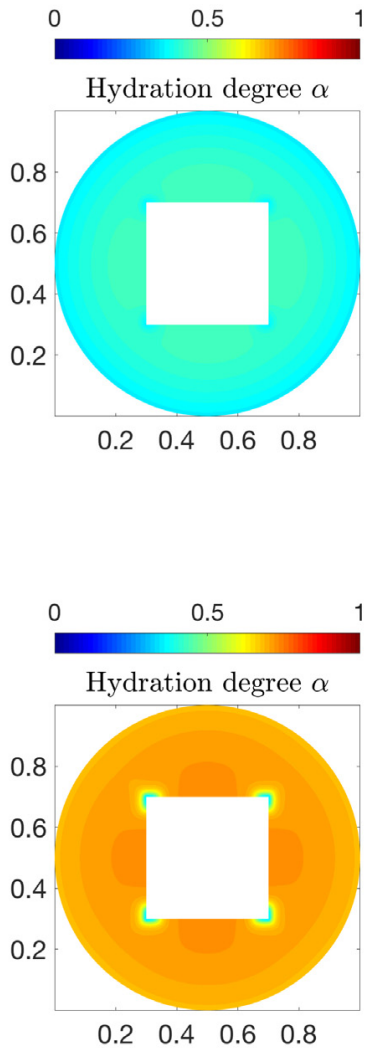

Fig. 4. The evolution of the phase field, temperature, and hydration of the structure during the hydration process calculated by the present approach The thermal expansion strain resulted in crack nucleation and then shrinkage strain led to the crack growth at the four vertices of the square hole.

diffused region. The incremental time step is taken as $\Delta t=300 \mathrm{~s}$ for the first $200 \mathrm{steps}$, and then $\Delta t=600 \mathrm{~s}$ for the total of 1500 time steps. The plane strain condition is assumed to this analysis.

The evolution of temperature, hydration degree and phase field during the hydration process is depicted in Figs. 4 and 5 and quantitatively detailed in Fig. 6 for the three investigated positions 1, 2 and 3 (as already described in Fig. 3). Basically, we observe three main periods of chemo-thermo-mechanical/fracture behaviors, which are described in each figure, and listed as follows:

(i) Time period $t=[0-25] \mathrm{h}$ : Hydration acceleration, along with the increase of heat until reaching its maximum, and the initiation of thermal crack. 

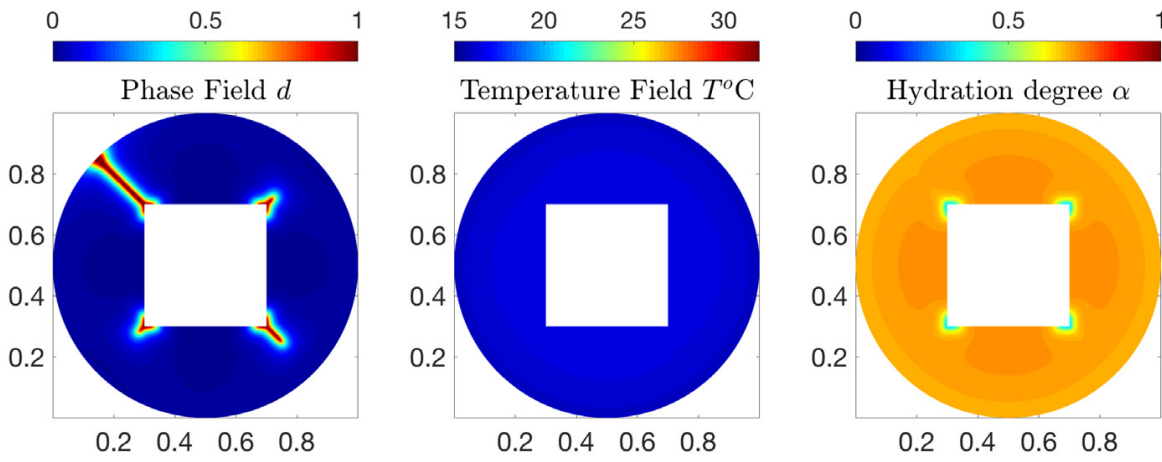

(a) time $t=118$ hours
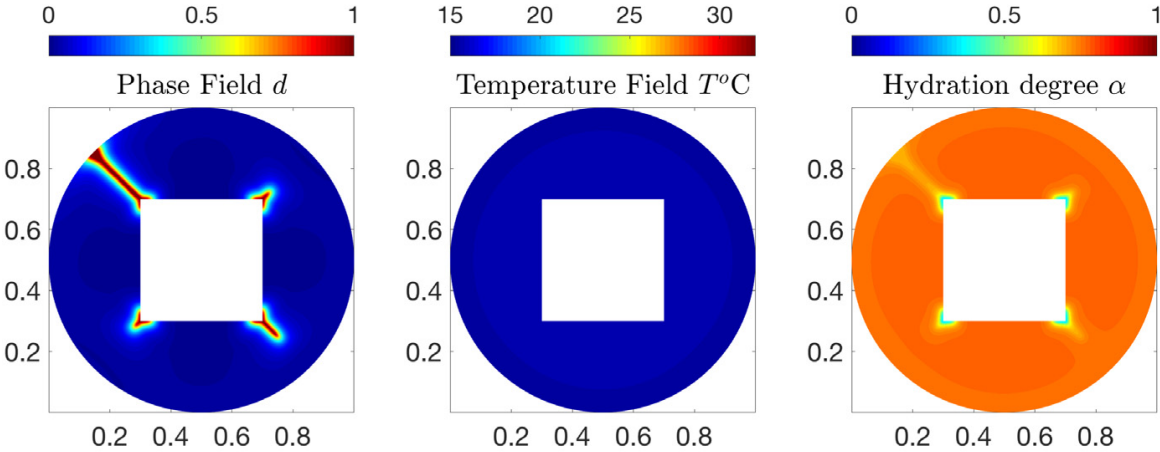

(a) time $t=200$ hours

Fig. 5. The evolution of the phase field, temperature, and hydration of the structure during the hydration process calculated by the present approach. The thermal expansion strain resulted in crack nucleation and then shrinkage strain led to the crack growth at the four vertices of the square hole.

(ii) Time period $t=(25-110] \mathrm{h}$ : Hydration deceleration, along with the decrease of heat, and the development of shrinkage strain inducing the switching of fracture cause mode from thermal crack mode to shrinkage crack mode.

(ii) Time period $t>110 \mathrm{~h}$ : Hydration deceleration and the crack growth through the investigated domain.

More specifically, in the first period (i) with $t=[0-25] \mathrm{h}$, the rapid increase of the hydration degree (hydration acceleration period) provided the released hydration heat that is greater than the heat loss due to convection to the environment. Hence, it resulted in the increase of temperature, reaching to a maximum temperature of $T \approx 33{ }^{\circ} \mathrm{C}$. The nucleation of crack (mainly due to the thermal expansion deformation) at four vertices in the square hole is then observed as sketched in Fig. 4(a). The second period (ii), with $t=(25-110] \mathrm{h}$ corresponds to the period of hydration deceleration, along with the decrease of temperature (the released hydration heat here is smaller than the convective heat loss, see Fig. 6(a)). It seems that the cracks stop growing during the second period. This phenomenon could be explained by the strength development of cement material thanks to the hydration process. As depicted in Fig. 7, the material reaches closest to $70 \%$ of its strength (i.e., Young's modulus and fracture resistance), which would be sufficient to resist the thermal and shrinkage strain.

However, in the third period (iii), with $t=[110-118] \mathrm{h}$, the development of the shrinkage strain causes the increase of tensile stress at the vertices, ${ }^{5}$ which exceeds the material strength (or material resistance). Hence, the crack propagation is then captured as shown in Figs. 4(b), 5(a). The fracture almost grows at the two vertices. Finally, at one

\footnotetext{
5 This phenomenon will be shown in Fig. 10 and corresponded text in the interpretation of mechanical behavior.
} 


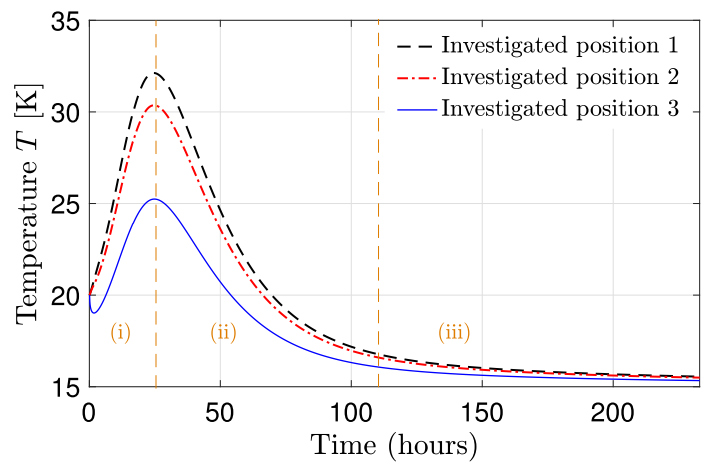

(a) Temperature evolution

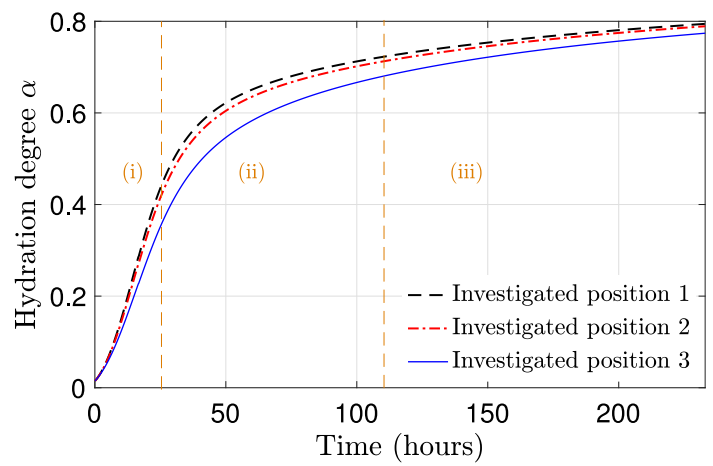

(b) Hydration evolution

Fig. 6. Temperature/hydration evolution at different positions in the solid domain. The structure reaches the maximum temperature at time $t=25 \mathrm{~h}$ and then decreases in time. The increase of temperature provided a quick hydration period for time $t<25 \mathrm{~h}$.
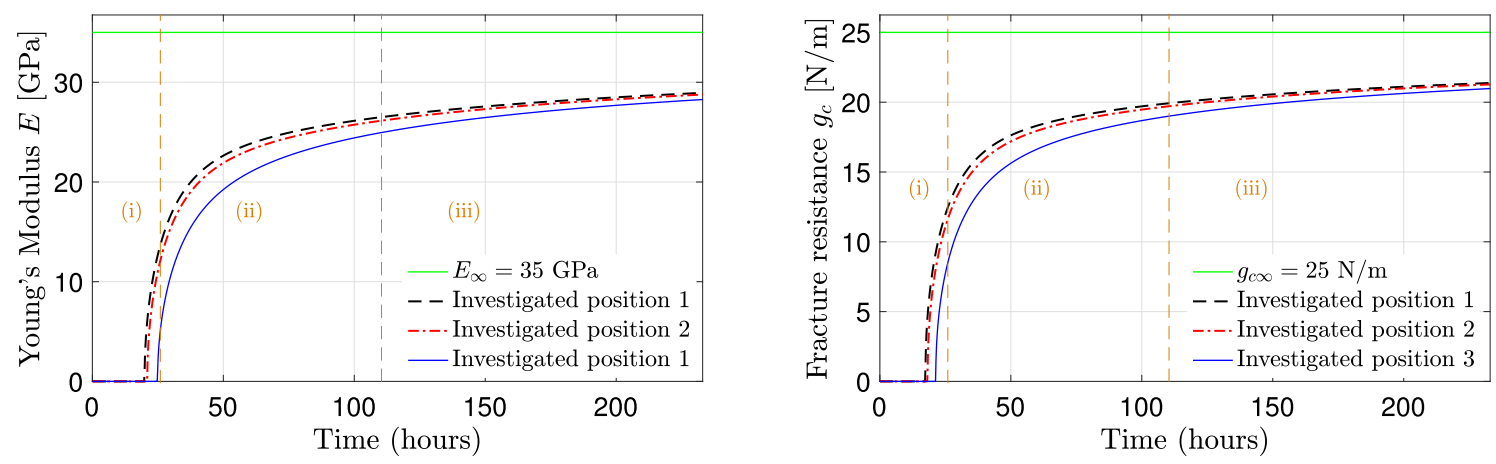

Fig. 7. The development of the Young's modulus and fracture resistance at three investigated positions. The material initiates its strength at time $t=20 \mathrm{~h}$ when the hydration degree $h \geq 0.35$ and obtains $80 \%$ of its maximum value after 10 days.

vertex, the crack totally propagates through the solid. The antisymmetric fracture phenomena found are probably due to the non-symmetric mesh of investigated structure and/or small incorrect symmetrical geometry. This error provides the antisymmetric stress distribution and then crack in one vertex will prevail on the other one. The stress is relaxed when the crack propagates through the considered domain. Moreover, the material has reached nearly $80 \%$ of its strength. Hence cracks do not develop any more as exhibited in Fig. 5(b) for time $t=200 \mathrm{~h}$.

The cracks significantly alter the mechanical behavior of structure and reduce its resistance. To better understand insight into this situation, we thus exhibit the evolution of elastic strain and energy during the whole hardening process. More specifically, the $\varepsilon_{y y}$ component of the averaged elastic strain at the upper-end of square hole $(y=0.7 \mathrm{~m})$, and the global elastic/fracture/total energies of the whole domain are plotted in Figs. 8 and 9, respectively. Three elastic strain evolution's stages are also observed according to the three aforementioned periods (i), (ii), (iii). Within the period $t=[0-25] \mathrm{h}$, the strain firstly increases thanks to the rising temperature produced by hydration reaction (strain is in a tensile state). Then, the hydration process goes into next step, in which the autogenous shrinkage strain prevails on thermal expansion strain, induced the decrease of strain. The elastic energy and total energy quickly grow in this period. The thermal crack initiation also provides a development of fracture-surface energy as represented in Fig. 9. The second period (ii), with $t=(25-110] \mathrm{h}$, captures the decrease of strain, turning into the compression state and switching the fracture cause from thermal cause mode to shrinkage cause mode. These phenomena are clearly demonstrated in the evolution of elastic and total energies, where the changing of fracture cause takes place at time $t \approx 50 \mathrm{~h}$. The final period (iii), with $t=(110-250] \mathrm{h}$, corresponds to the crack growth period. The local distribution of all three strain components are plotted in Fig. 10 at time $t=112 \mathrm{~h}$. The shear mode at four vertices is observed, and will be a main cause of shear fracture propagation. With the crack growth, the elastic strain component $\varepsilon_{y y}$ relaxes 


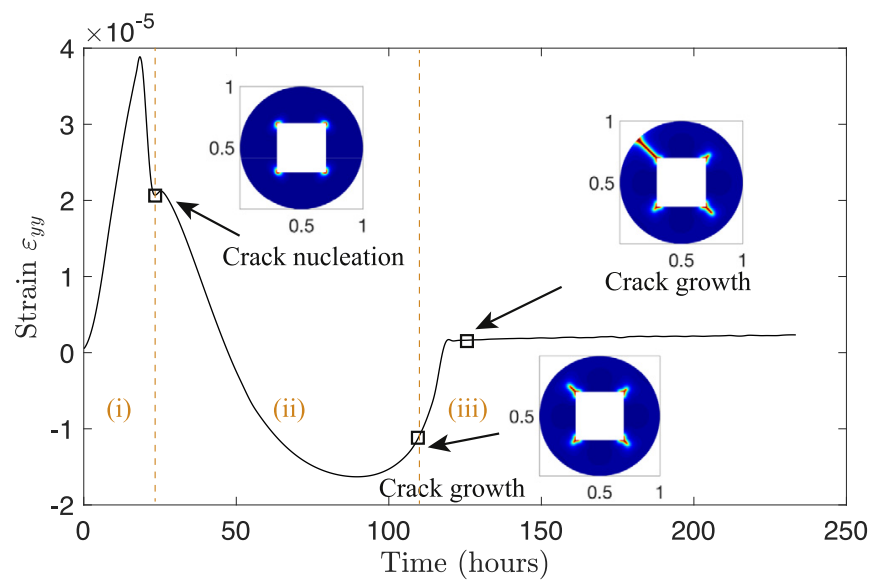

Fig. 8. The evolution of overall elastic strain during hydration process, three periods have been observed: (i) tension period due to the increase of temperature and then (ii) reducing cause of shrinkage process (iii) relaxing period corresponding to crack nucleation/propagation.

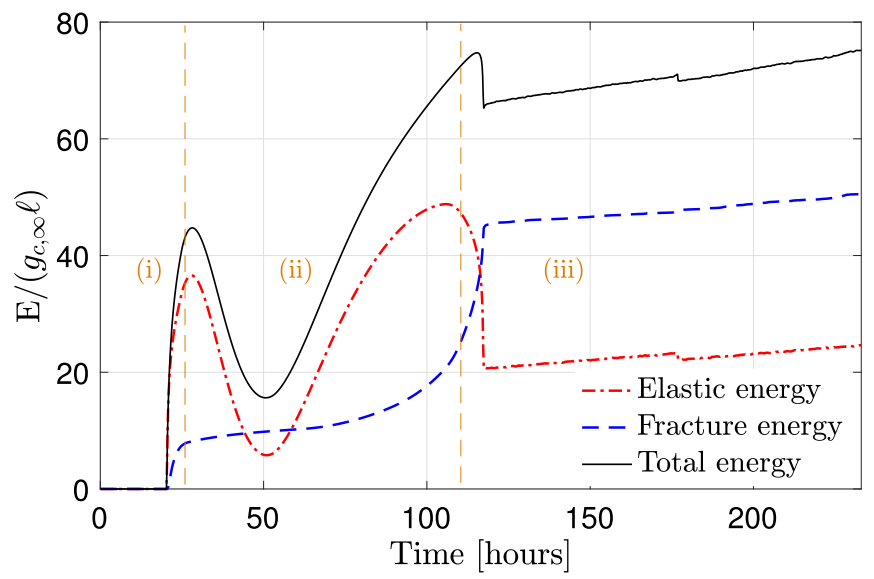

Fig. 9. The evolution of global elastic energy, fracture-surface energy and total energy during the hydration process.

very quickly, and turns into a completely relaxed state at time $t \approx 125 \mathrm{~h}$ once the crack propagates through the solid completely.

The extremely complex chemo-thermo-mechanical and fracture behaviors of concrete structure during the hydration process are captured by the present model. The hydration process, along with thermal transfer proceeds in multi-stages, and induces the competition between the thermal expansion strain and the autogenous shrinkage strain. These phenomena strongly affect the mechanical performance of cement-based materials, which has been successively simulated by the proposed model via the prediction of different cracking stages, such as thermal crack initiation and shrinkage crack propagation. Obviously, an accurate and efficient computational model, which possibly predicts this complex behavior would be helpful for material design in engineering applications.

\subsection{Cement-based microstructure: hydration reaction induced fracture analysis}

The main purpose of this numerical example is to illustrate the capability of the developed approach in solving problems with complex propagation in real material obtained from microtomography of an lightweight concrete. The considered 2D microstructure is a cross-section of a microtomography image of a real cementitious material obtained by the XRCT laboratory scanner available at Navier laboratory [53]. The real material consists of coarse sand embedded into a cement matrix. The gray level image was filtered and thresholded to separate the two phases 

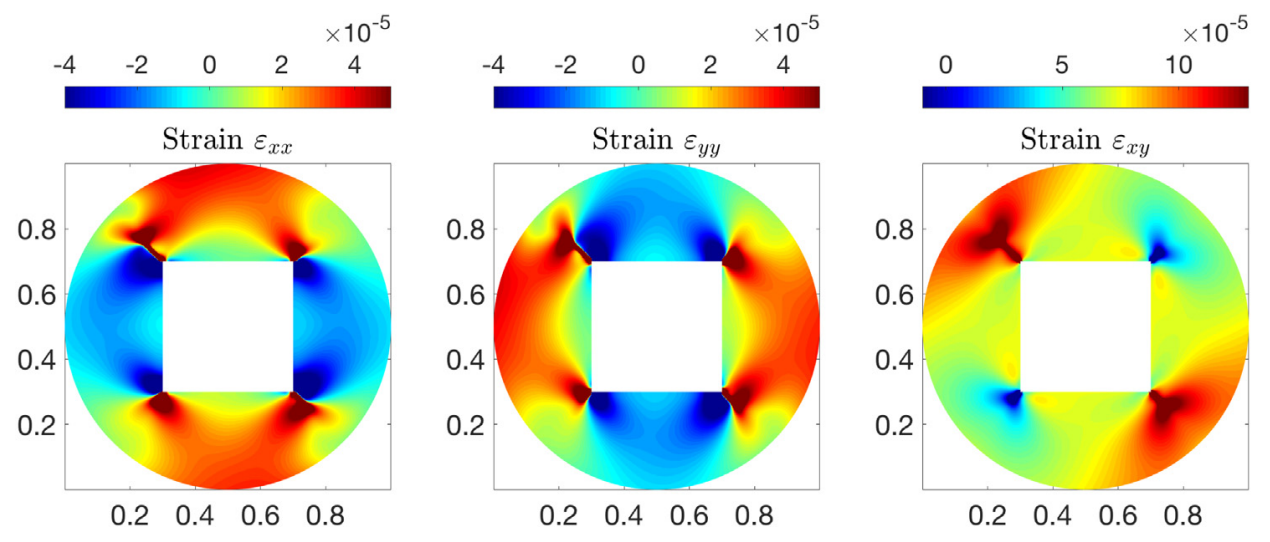

Fig. 10. The local distribution of elastic strain field at time $t=112 \mathrm{~h}$.
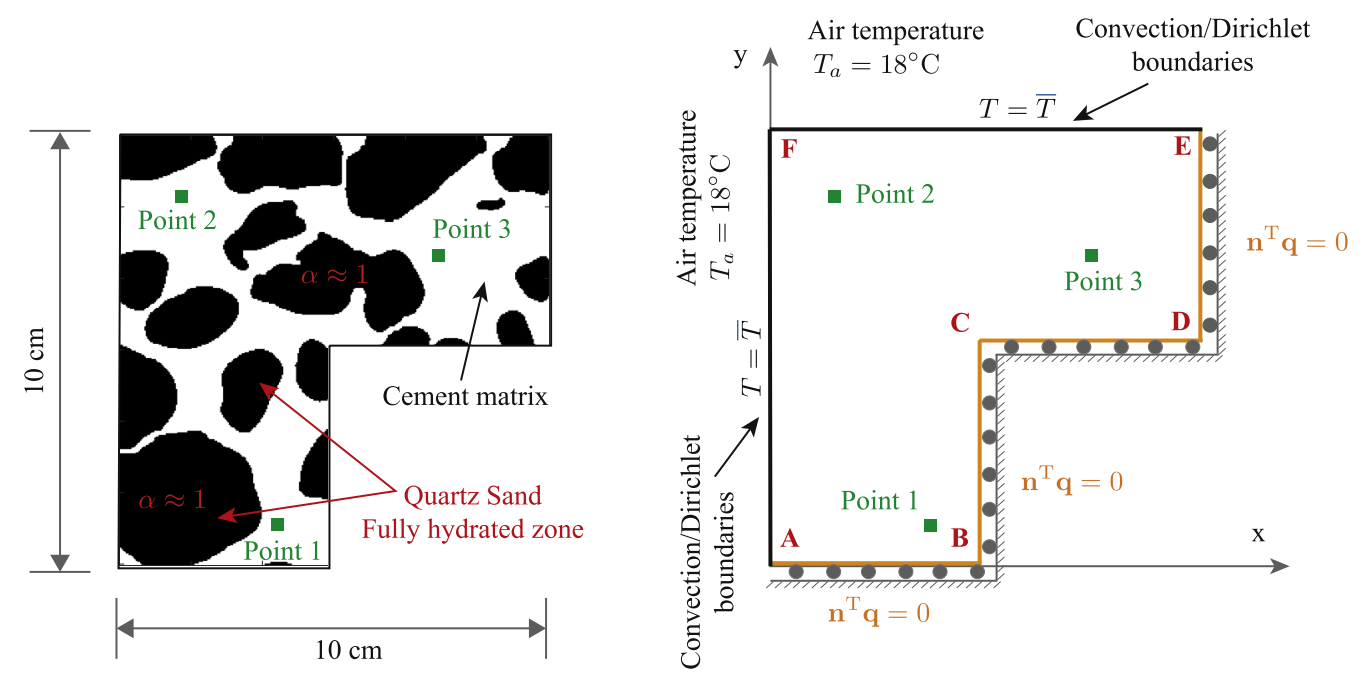

Fig. 11. Geometry and boundary conditions of an investigated structure, where the microstructure is explicitly represented by using X-ray tomography image.

of the microstructure. The computed results are then represented in Fig. 11(a), where the white and black phases correspond to the matrix (cement paste), inclusions (sand grains), respectively.

The boundary conditions and geometry of the sample are also depicted in Fig. 11(b). The Dirichlet boundary condition $T=\bar{T}$ and radiation/convection conditions are applied on the left-end AF $(x=0)$ and upper-end EF $(y=10 \mathrm{~cm})$. The displacements in both $x, y$-directions of these two edges AF and EF are here kept to be free. The four edges $\mathrm{AB}, \mathrm{BC}, \mathrm{CD}, \mathrm{DE}$ with orange color are subjected to zero flux condition, with the displacement's constraint that are defined as follows: on the edge $\mathrm{AB}$ and $\mathrm{CD}$, displacements in $y$-direction are fixed while displacements in $x$-direction are free; on the edge BC and DE, displacements in $x$-direction are fixed while displacements in $y$-direction are free. The switching between the convection and Dirichlet boundary conditions in time are described in Table 2. The use of these mixed boundary conditions is twofold: (i) demonstrating the robustness of the proposed model and its numerical implementation; (ii) highlighting the effects of conducting crack and insulating crack on the multi-physics problem of cement hydration. In particular, it proves the capacity of the proposed numerical scheme in dealing with a 
Table 2

Description of boundary conditions.

\begin{tabular}{llll}
\hline Period & $t=[0-13.5] \mathrm{h}$ & $t=(13.5-72] \mathrm{h}$ & $t>72 \mathrm{~h}$ \\
\hline Left-end AF & Dirichlet $\bar{T}=35^{\circ} \mathrm{C}$ & Convection $T_{a}=18{ }^{\circ} \mathrm{C}$ & Convection $T_{a}=18{ }^{\circ} \mathrm{C}$ \\
Upper-end EF & Dirichlet $\bar{T}=35^{\circ} \mathrm{C}$ & Convection $T_{a}=18{ }^{\circ} \mathrm{C}$ & Dirichlet $\bar{T}=100^{\circ} \mathrm{C}$ \\
\hline
\end{tabular}

Table 3

Material properties.

\begin{tabular}{llllllll}
\hline Parameter & Cement & Sand & Unit & Parameter & Cement & Sand & Unit \\
\hline$\rho c$ & 2400 & 2000 & $\mathrm{~kJ} /\left(\mathrm{K} \mathrm{m}^{3}\right)$ & $\alpha_{a u}$ & 0.115 & 0.115 & - \\
$k$ & 2.8 & 0.75 & $\mathrm{~W} /(\mathrm{m} \mathrm{K})$ & $\alpha_{E}$ & 0.35 & 0.35 & - \\
$Q_{\infty}$ & 117840 & 117840 & $\mathrm{~kJ} / \mathrm{m}^{3}$ & $\alpha_{g_{c}}$ & 0.25 & 0.25 & - \\
$h$ & 8 & 8 & $\mathrm{~W} /\left(\mathrm{m}^{2} \mathrm{~K}\right)$ & $\ell$ & 0.85 & 0.85 & $\mathrm{~mm}$ \\
$\beta$ & 7.5 & 3 & $\mu \mathrm{m} /(\mathrm{m} \mathrm{K})$ & $\nu$ & 0.22 & 0.2 & - \\
$\kappa$ & 40 & $10^{-10}$ & $\mu \mathrm{m} / \mathrm{m}$ & $T_{0}$ & 20 & 20 & ${ }^{\circ} \mathrm{C}$ \\
$E_{\infty}$ & 35 & 72 & $\mathrm{GPa}$ & $A_{T}$ & 0.215 & 0.215 & $\mathrm{GW} / \mathrm{kg}$ \\
$g_{c}$ & 5 & 250 & $\mathrm{~N} / \mathrm{m}$ & $E_{a}$ & 45.93 & 45.93 & $\mathrm{~kJ} / \mathrm{mol}$ \\
\hline
\end{tabular}

very complex heat transfer condition. ${ }^{6}$ Furthermore, this set-up is employed for simulating the case when the cementbased materials are drying in several regions, and for especially studying the manufacture of the concrete element with some heating procedure, e.g., a fiber reinforced concrete using shape memory alloys (requiring a heat treatment).

From the $500 \times 500$ pixels, a mesh of $\approx 80000$ triangular elements is generated, where the material property of matrix and inclusion are transferred into the triangular domains associated with elements. A detailed description was previously reported by the present authors, and curious readers should refer to, see e.g., [22] for more information.

The material parameters are detailed in Table 3. The Young's modulus of sand grains, $E_{\infty}=72 \mathrm{GPa}$, is chosen twice larger than that of the cement matrix, i.e., $E_{\infty}=35 \mathrm{GPa}$. The internal length is specified as homogeneous for both cement and sand, using Eq. (44) with the tensile strength of sand $\sigma_{c}=45.3 \mathrm{MPa}$, we thus obtain $\ell \approx 1 \mathrm{~mm}$. The sand grain is assumed to be totally hydrated $\alpha_{\text {sand }}\left(t_{0}\right) \approx 1$ (in other words, the quartz sand is not part of the hydration process). This definition essentially leads to chemical affinity $f\left(\alpha_{\text {sand }}\right)=0$ during the whole simulation. It means that there is no heating producing in the sand region, which is linked to the fact that no chemical reaction between sand and water takes place. The hydration threshold, in which material starts having the strength is selected the same for both phases. It is important to notice that quartz sand is already fully hydrated. Hence its material strength reaches the maximal value at the beginning of the hydration process and does not provide any autogenous shrinkage strain, to ensure that, $\kappa_{\text {Sand }} \approx 0$ is chosen. Moreover, for the sake of simplicity, the special behaviors of the interfacial transition zone are not considered here. Two situations of thermal transfer passing cracked area are investigated. In the first case (C1), cracks are conducting, i.e., $\xi=0$, and the second case (C2) when the cracks are insulating, we set $\xi=1$.

The computed results of crack growth, temperature, and hydration evolution for the first case of conducting crack (C1) are depicted in Fig. 12. It should be noticed here that a post-processing procedure is adopted to highlight the effects of quartz sand on the fracture problem. The geometry of sand grains are represented and marked in black color and used as a background, which is then overlaid with the phase field. The development of temperature and hydration degree at three typically investigated positions (IP1,2,3) is also shown in Fig. 13. In general, we capture three different chemo-thermo-mechanical/fracture behaviors' periods, corresponding to the three periods of the switching boundary conditions. In the first period (i) with $t=[0-13.5] \mathrm{h}$, the thermal effects play a major role that alter the material behavior. More specifically, the different thermal behavior between cement and sand provides a heterogeneous temperature distribution and hydration process. It can be seen that the sand grains are deformed less than cement, providing the stress concentration at interfacial region. At the time, e.g., $t \approx 8.33 \mathrm{~h}$, the damage nucleation can be observed at several interfaces of sand/cement as represented in Fig. 12(a). The occurrence of damage in this period is the consequence of the thermal expansion, due to thermal transfer from boundaries and hydration process. More interestingly, these damages do not develop due to the rapid strength increase at that period. The material strength

\footnotetext{
6 The used boundary conditions also mimic the experimental conditions described in [54]. This issue will be clarified in the comparison with experiential observation.
} 


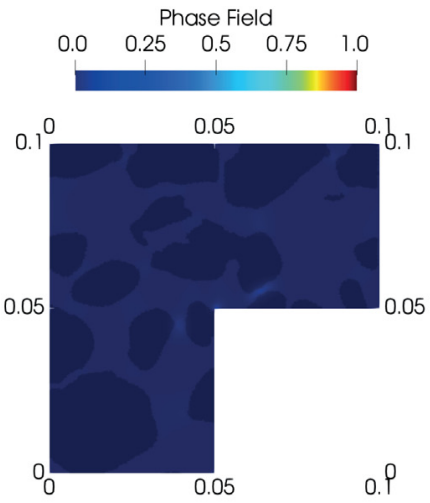

(a) $\mathrm{t}=8.33$ hours

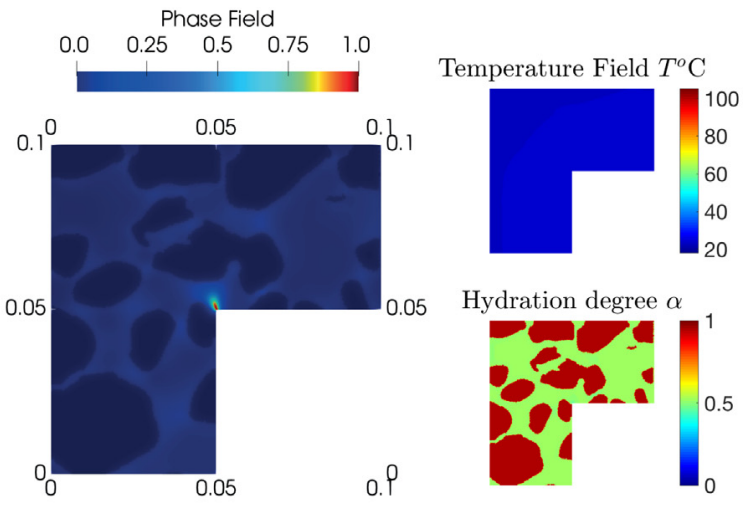

(b) $\mathrm{t}=18.16$ hours

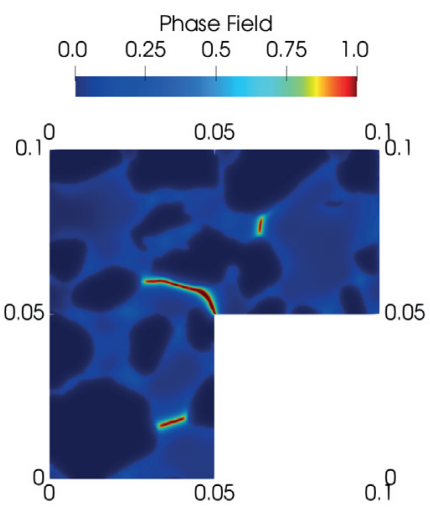

(c) $t=55.83$ hours
Temperature Field $T^{\circ} \mathrm{C}$
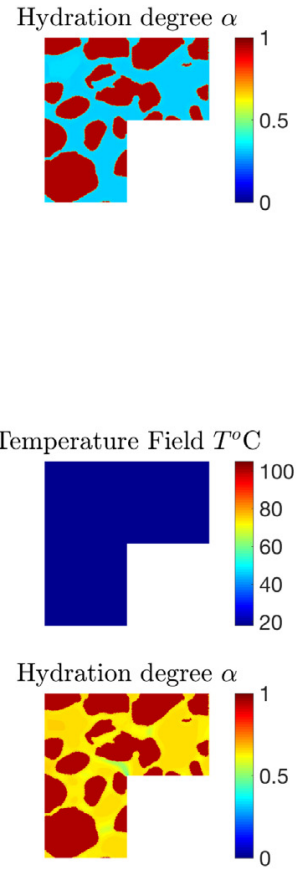

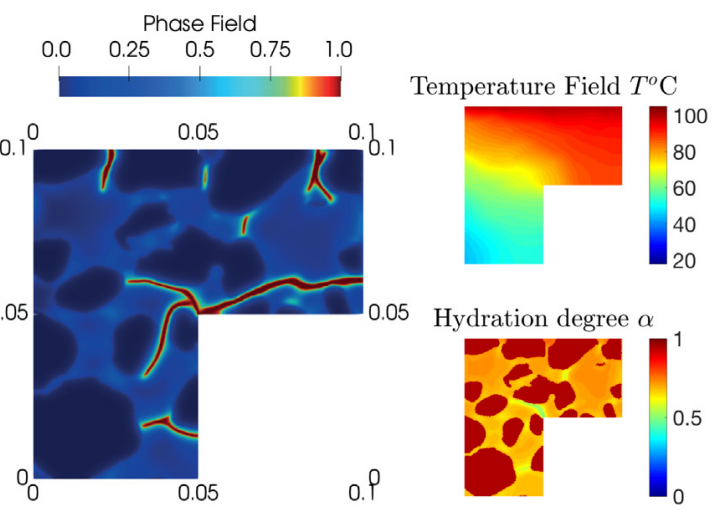

(d) $t=73.36$ hours

Fig. 12. Conducting crack $(\mathrm{C} 1)$ : the evolution of phase field, temperature and hydration of the structure at different time steps. Together with the material hardening (increasing of hydration degree), the crack initiation and propagation are observed.

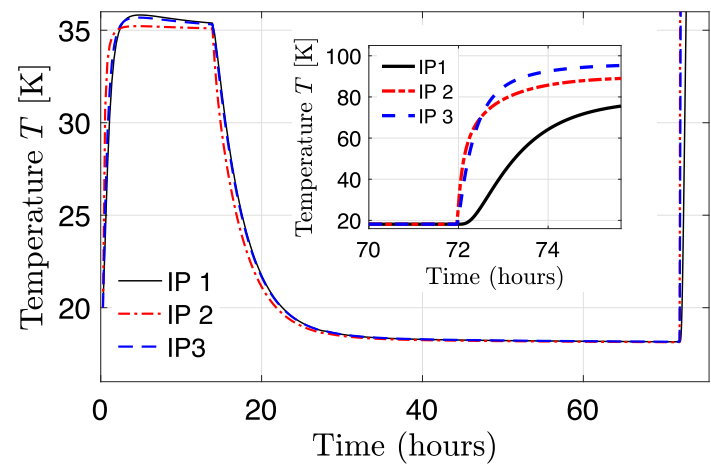

(a) Conducting crack (C1)

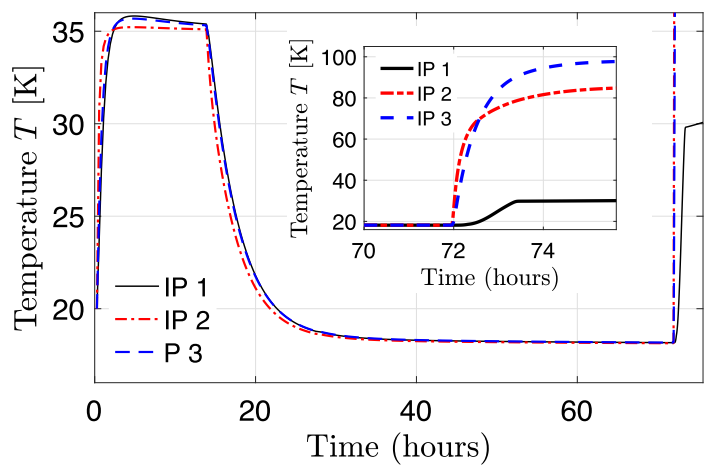

(b) Insulating crack $(\mathrm{C} 2)$

Fig. 13. The evolution of temperature during the hydration process obtained by the present approach. Three big gap evolution is represented corresponding to (i) set to prescribed temperature $\bar{T}$, (ii) switch to convection condition, and (ii) reset again to Dirichlet boundary condition. The difference of temperature level at the investigated point 1 between two cases is clearly observed. 

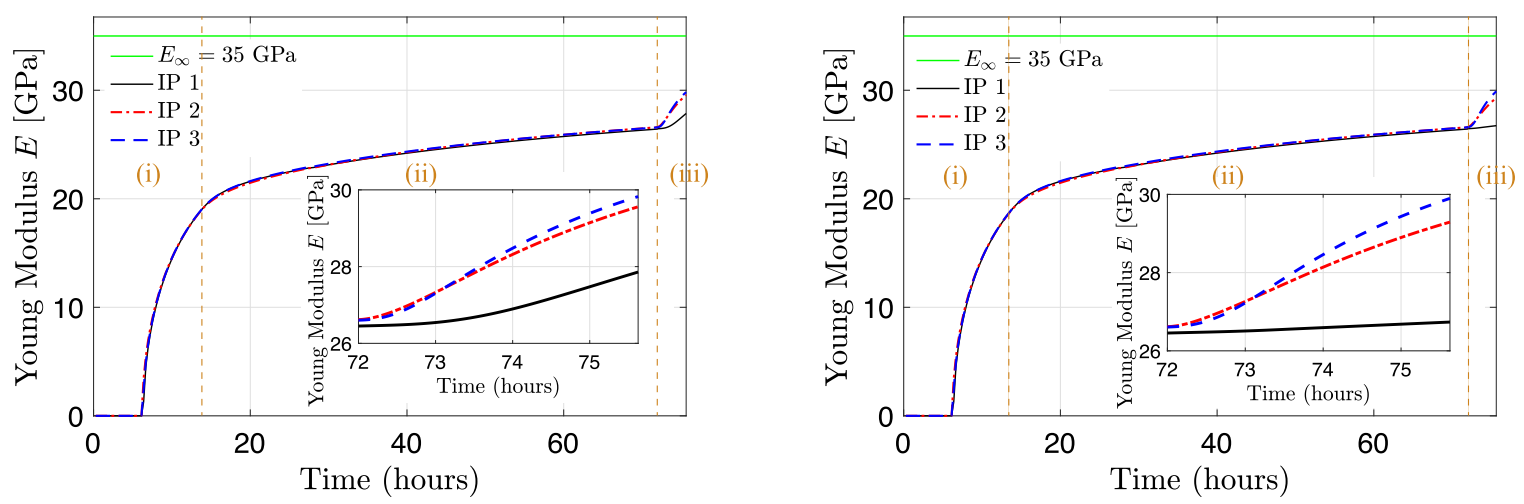

Fig. 14. The development of the Young's modulus and fracture resistance for the three investigated positions during the hardening process for the case of conducting crack (C1). The material initiates its strength at time $t=7 \mathrm{~h}$ when the hydration degree $h \geq 0.25$ and gets almost $80 \%$ of its maximum strength after 7 days.

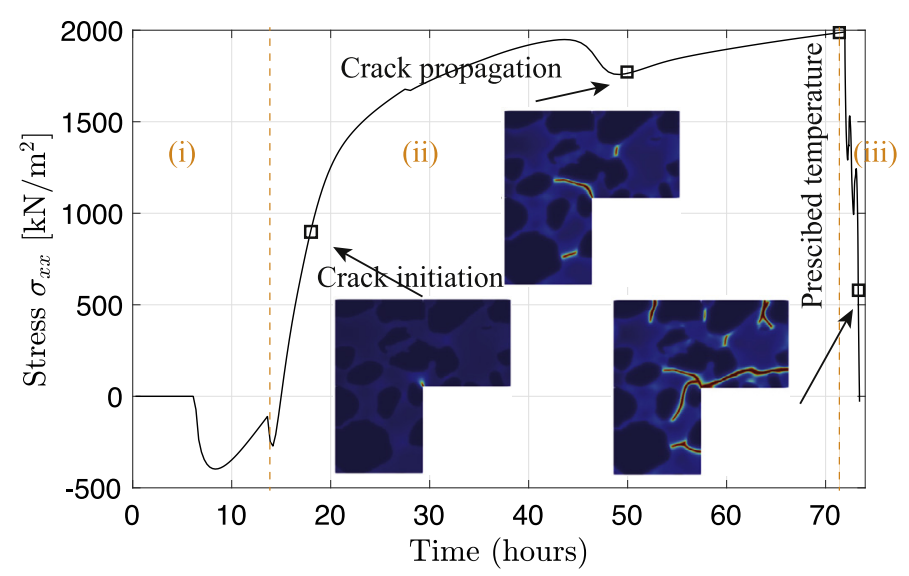

Fig. 15. The evolution of averaged stress $\sigma_{x x}$ at the edge $x=5 \mathrm{~cm}$.

almost reaches their 50\% maximum value after a period of $13.5 \mathrm{~h}$, as depicted in Fig. 14. The hardening in this example gets is much more rapid than that of the previous example thanks to the prescribed temperature, which accelerates the hydration process. However, in the second period (ii), without the prescribed temperature, the autogenous shrinkage strain now pilots the behavior of the investigated structure. At the time $t>13.5 \mathrm{~h}$ the shrinkage strain increases very quickly and then provides the stress concentration beyond material strength at the vertex of the investigated structure. The crack initiation is observed at the time $t=18.16 \mathrm{~h}$ (see Fig. 12(b)) corresponding to a hydration degree of $\alpha \approx 0.55$. The crack onset leads to the nonlinear evolution of stress, as captured in Fig. 15, where the averaged stress at the edge $\mathrm{BC}(x=5 \mathrm{~cm})$ is plotted. The crack propagation clearly reduces the material resistance. A gap of stress evolution is observed at time $t=45 \mathrm{~h}$, related to the occurrence of two new cracks and propagation of crack at the vertex. In the third period, the thermal shock due to the prescribed temperature at time $t=72 \mathrm{~h}$ provides a significant changes in the fracture problem. A complex crack network is obtained at time $t=73.36 \mathrm{~h}$ in Fig. 12(d), inducing a big drop of material resistance within this period, Fig. 15.

Fig. 16 presents the results for the second case (C2) of the insulating crack. The evolution of phase field, temperature and hydration degree is quasi the same as the previous situation (C1) for the two time periods (i),(ii), or with $t=[0-72] \mathrm{h}$. The significant difference is captured for $t>72 \mathrm{~h}$ when the system is subjected to the second Dirichlet boundary condition (prescribed temperature). In the case of conducting crack, the thermal transfer is not affected by the crack, see Fig. 17(a). Contrarily, the insulating crack does not allow thermal transfer crossing cracked region. Therefore a big gap of temperature distribution is captured between two sides of the crack, as depicted in Fig. 17(b). The different temperature distribution between two cases will directly affect the hydration process. 


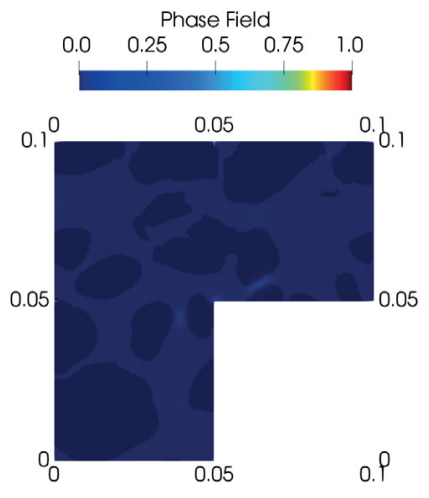

(a) $\mathrm{t}=8.33$ hours

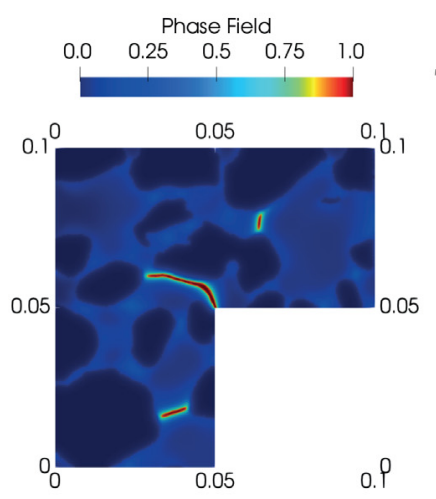

(c) $\mathrm{t}=55.83$ hours

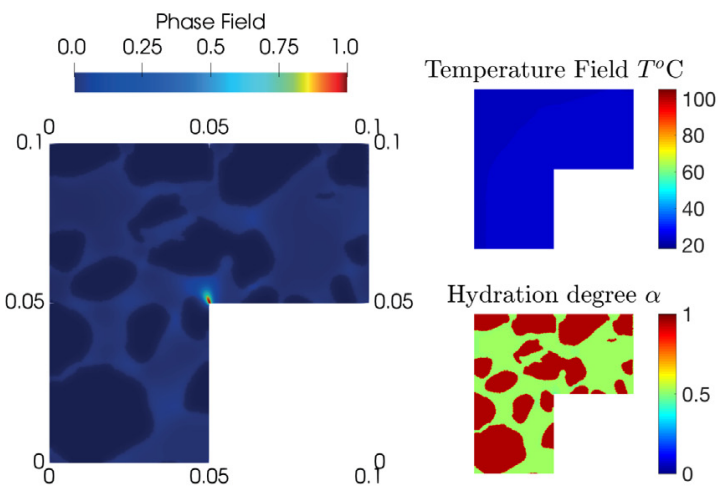

(b) $\mathrm{t}=18.16$ hours
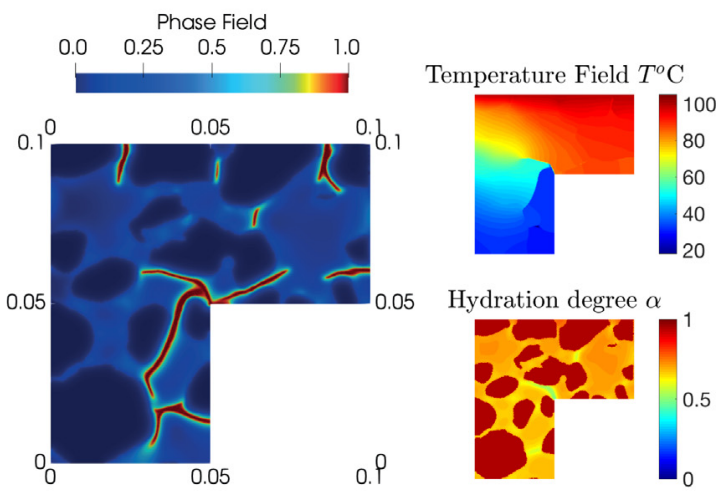

Hydration degree $\alpha$
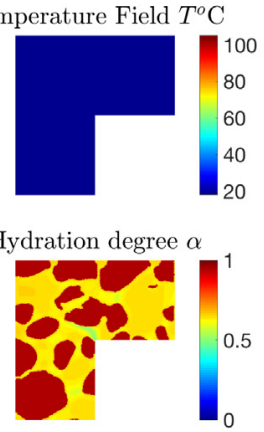

(d) $\mathrm{t}=73.36$ hours

Fig. 16. Insulating crack (C2): The evolution of phase field, temperature, and hydration of considered structure at different time steps. Together with the hardening of material (increasing of hydration degree), the crack initiation and propagation are observed.

The conducting crack resulted in a higher temperature on the left side of the crack, which is suitable for hydration rate. These phenomena are clearly observed in Fig. 18, since for $t>72 \mathrm{~h}$ (time period (iii)), the hydration degree of insulating crack situation is less than that one of the conducting crack case. Different fracture phenomena are captured. The adiabatic fracture scenario (C2) provides a weak region close to the edge $\mathrm{BC}(x=5 \mathrm{~cm})$, as its temperature/hydration degree is much smaller than that at other regions. Hence, the crack propagation has occurred at this zone. It is different to the case $(\mathrm{C} 1)$, where fracture is more dominant at the zone close to the edge $\mathrm{CD}(y=5 \mathrm{~cm})$, see Fig. 17.

The obtained numerical results shed light on the important role of fracture in the hydration process. The occurrence of damage/crack will modify the material properties, influencing on thermal transfers, altering the hydration evolution and impacting the mechanical behavior of structures and materials.

Finally, in order to qualitatively verify the prediction of the numerical model, a comparison of crack morphologies between experimental observation reported by Wong et al. [54]) and numerical simulation by the present model in Fig. 19 is provided. The numerical simulation has been performed in similar conditions compared to the experiment. ${ }^{7}$ Three periods of thermal boundary conditions described in Table 2 mimic the experimental conditions: the first period (i) corresponds to the drying period, the second period (ii) corresponds to the transport tests (oxygen diffusivity, oxygen permeability and water sorptivity), and the third period (iii) corresponds to the heating conditions in the

\footnotetext{
7 In the experimental data of Wong et al. [54], the tomography image only provided the fracture behavior of a sub-region of the investigated structure. The thermo-mechanical boundary conditions of this sub-zone are unknown. In the numerical model, we also performed the simulation on a small region and tried to mimic maximum the experimental boundary conditions.
} 

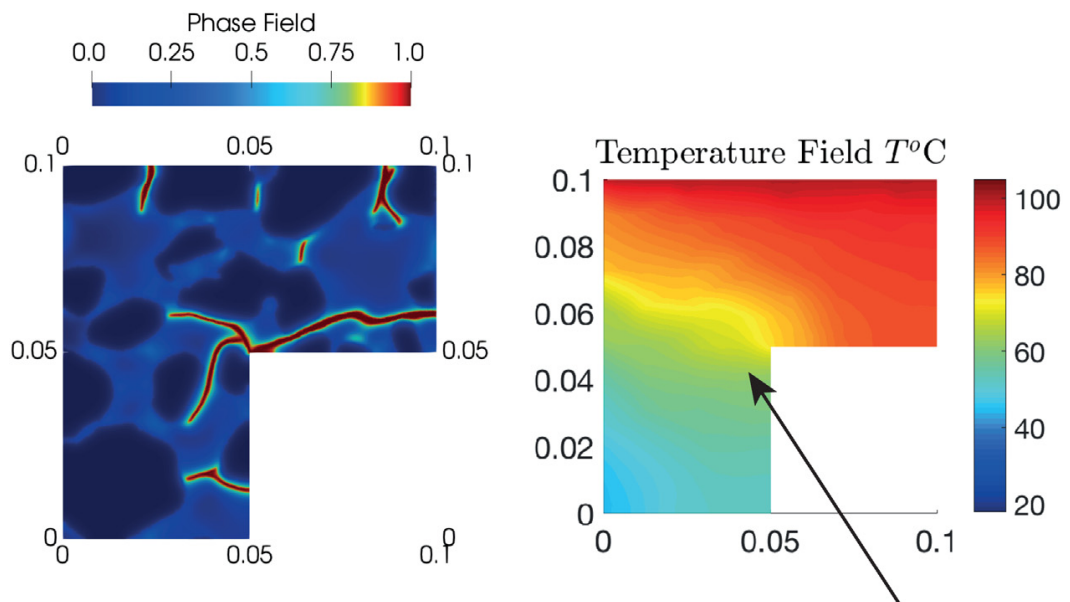

(a) (C1) Conducting crack: time $t=73.36$ hours
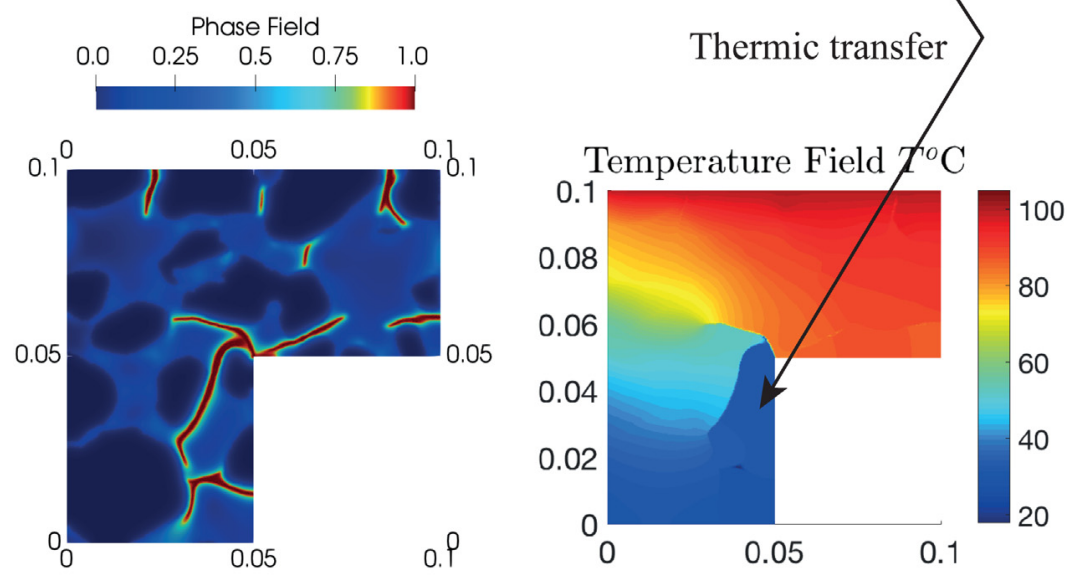

(b) (C2) Insulating crack: time $\mathbf{t}=73.36$ hours

Fig. 17. Comparison of the fracture behavior and the thermal transfer between (a) conducting crack (C1), and (b) insulating crack (C2).

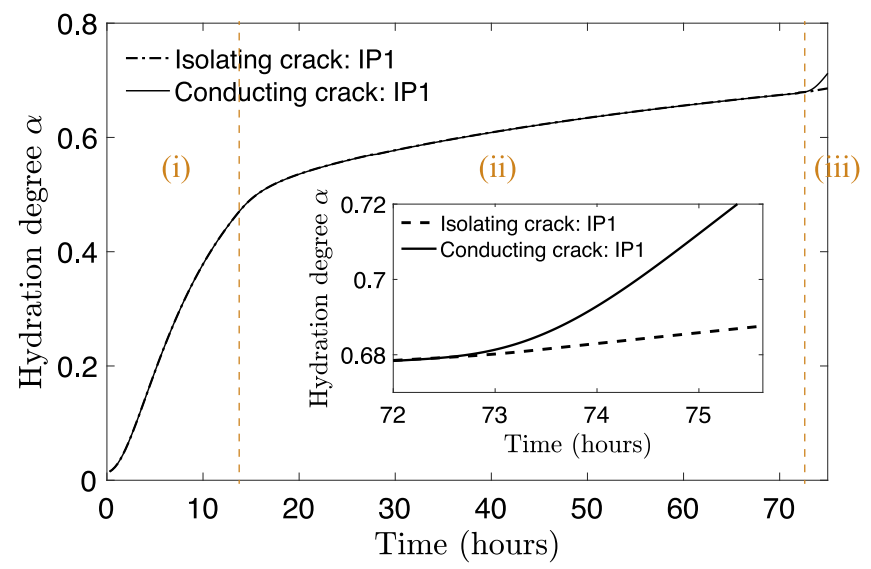

Fig. 18. The development of hydration degree during hardening process, a comparison between the conducting crack (C1) and insulating crack (C2). The conducting crack (C1) seems to accelerate the hydration process. 


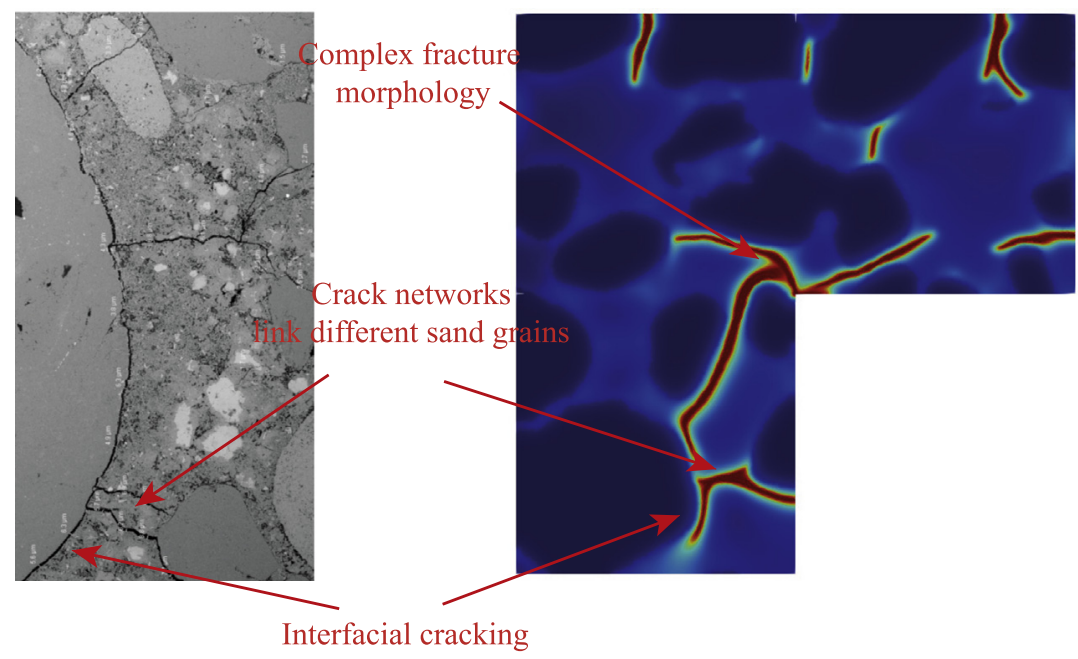

(a) Experimental observation

(b) Numerical prediction

Fig. 19. A comparison of crack evolution between experimental characterization reported in Ref. [54] and numerical prediction by the present phase field model. Several important aspects of hydration induced crack propagation in experiment are reproduced by simulation, such as interfacial cracks, crack linking different grains.

experiment. The experimental characterization using Backscattered electron microscopy captured a complex fracture network. The cracks take place at the interfacial region and they have a propagation tendency to link different sand grains, as depicted in Fig. 19. The developed model qualitatively predicts these fracture phenomena. It captures several important aspects of experimental findings, such crack propagation following interfaces of cement/sand and creating a complex crack network among different sand grains. The provided comparisons are still in a phenomenological way, due to the lack of experimental data regarding microstructural details, boundary conditions, and material parameters. Further explorations and validations (quantitative comparison by using e.g., multi-scale simulations) of this conjecture would be an interesting subject and should be performed in future works.

\section{Conclusions}

In this paper, we developed a new multiphysics framework based on phase field model for simulating complex fracture mechanism induced by the heat of hydration and shrinkage strain at early-age. This contribution of numerical simulation is a starting point that models the hydration process by using a chemo-thermo-mechanical coupling scheme. The proposed model efficiently simulates the heat of hydration, thermal transfer and the material strength's development thanks to age effects. These processes are incorporated into the phase field framework of regularized crack to examine fracture behavior of the structures and materials. The most important interactions among chemothermo-mechanical and fracture processes are included in the present model. Specially, the effects of cracked area on hydration and thermal transfer are also taken into account. The numerical implementation is based on the staggered scheme (one-pass solution), by which three main processes such as the chemo-thermal problem, mechanical problem, and fracture problem are solved at each time step. The introduction of the staggered update scheme of time-discrete fields, together with the robust approximated formulation of the local history field provides: (i) one non-linear problem for the update of the temperature field, and the hydration field; (ii) two linear problems for the successive update of the phase field, and the displacement field within a typical time step. Hence, the proposed numerical scheme is extremely stable (i.e., the convergence of non-linear chemo-thermal problem often requires only 3-4 iterations). Moreover, thanks to the staggered solution scheme, the degrees of freedom for each partial problem (temperature/hydration, phase field, displacement field) are not collocated in FE implementations. Therefore, it increases the computational efficiency of the method, which is promising to investigate a complex problem in 3D.

We have performed analysis of fracture phenomena for two different structure types: one is of the homogeneous structure and the other is of the strongly heterogeneous structure. The complex crack nucleation and propagation are 
simulated in an efficient manner. In addition, the comparison of numerical prediction and experimental observation is also carried out. In general, the complex damage/crack morphologies captured in the experiment are qualitatively reproduced by the present model. This contribution not only provides an effective potential approach to study the shrinkage/hydration assisted crack propagation, but also shows potential applicability for practical engineering applications. Moreover, the current model could also be possible to reproduce 3D experimental results, such as 3D crack morphology defined by X-ray tomography at the microscopic scale.

\section{Acknowledgments}

The support this work enjoys from SeRaMCo project (Secondary Raw Materials for Concrete Precast Products), through the transnational cooperation projects in North-West Europe (Interreg NWE), NWE-320 is gratefully acknowledged.

\section{Appendix A. Radiation and convection using the Newton's cooling law}

Based on Ref. [55], the radiation/convection coefficient can be taken as a sum of convection coefficient, $h_{c}$, and radiation coefficient, $h_{r}$ :

$$
h=h_{c}+h_{r}
$$

in which the convection coefficient is defined to be dependent on the wind speed $v$, yielding

$$
h_{c}= \begin{cases}5.6+3.95 v & \text { for } v \leq 5 \mathrm{~m} / \mathrm{s} \\ 7 v^{0.78} & \text { for } v>5 \mathrm{~m} / \mathrm{s}\end{cases}
$$

and the radiation coefficient is written as follows:

$$
h_{r}= \begin{cases}4.8 c_{\text {emiss }}\left[1+0.015625\left(T_{a}-278.15\right)\right] & \text { for } T_{a}>278.15 \mathrm{~K} \\ 4.8 c_{\text {emiss }} & \text { for } T_{a}<278.15 \mathrm{~K}\end{cases}
$$

where $c_{\text {emiss }}$ is the emissivity of concrete, is often taken within the range $c_{\text {emiss }}=[0.85-0.95]$

\section{Appendix B. Basics of thermodynamics and evolution of phase field}

In this appendix, a crack phase field evolution law is derived, which guarantees one important aspect of the present approach, the irreversibility of the process. Without external micro forces, the Clausius-Duhem inequality can be written as follows:

$$
\mathcal{D}=\sigma: \dot{\varepsilon}^{e}-\dot{\psi}-S \dot{T}-\boldsymbol{q} \cdot \frac{\nabla T}{T} \geq 0,
$$

where $S$ is the entropy density.

Using the definition of free energy $\psi$ in Eq. (17), the local dissipation (B.1) can be rewritten as

$$
\mathcal{D}=\left(\boldsymbol{\sigma}-\frac{\partial \psi}{\partial \boldsymbol{\varepsilon}^{e}}\right): \dot{\boldsymbol{\varepsilon}}^{e}-\left(\frac{\partial \psi}{\partial T}+S\right) \dot{T}+\mathcal{A}_{d} \dot{d}+\mathcal{A}_{\alpha} \dot{\alpha}-\boldsymbol{q} \cdot \frac{\nabla T}{T} \geq 0
$$

where $\mathcal{A}_{d}=-\frac{\delta \psi}{\delta d}=-\frac{\partial \psi}{\partial d}+\nabla \cdot\left(\frac{\partial \psi}{\partial \nabla d}\right)$ is the variational derivative of $\psi$ with respect to the phase field $d$. The chemical affinity $\mathcal{A}_{\alpha}=-\frac{\partial \psi}{\partial \alpha}$ is here identified the thermodynamic force associated in the (chemical) dissipation to the reaction rate $\dot{\alpha}$.

The thermodynamic restriction (B.2) can be spitted up into three parts: one due to local actions, one due to the chemical reaction and the last one related to heat conduction

$$
\begin{aligned}
& \mathcal{D}_{1}=\left(\sigma-\frac{\partial \psi}{\partial \boldsymbol{\varepsilon}^{e}}\right): \dot{\boldsymbol{\varepsilon}}^{e}-\left(\frac{\partial \psi}{\partial T}+S\right) \dot{T}+\mathcal{A}_{d} \dot{d}, \\
& \mathcal{D}_{2}=\mathcal{A}_{\alpha} \dot{\alpha}, \\
& \mathcal{D}_{3}=-\boldsymbol{q} \cdot \frac{\nabla T}{T},
\end{aligned}
$$

and the positive of $\mathcal{D}$ will be obviously ensured if $\mathcal{D}_{1}, \mathcal{D}_{2}$ and $\mathcal{D}_{3}$ are positives. 
In the case that the hydration rate is described by Arrhenius law in Eq. (6) and the thermal transfer problem is conducted by the Fourier's law, with $\boldsymbol{q}=-\boldsymbol{k} \nabla T$, the positive restriction for $\mathcal{D}_{2}$ and $\mathcal{D}_{3}$

$$
\mathcal{D}_{2}=\mathcal{A}_{\alpha} \dot{\alpha}=g(d)\left(\mathcal{A}_{\alpha}\right)^{2} e^{\left(\frac{-E_{a}}{R T}\right)} \geq 0 \text { and } \mathcal{D}_{3}=-\mathbf{q} \cdot \frac{\nabla T}{T}=\frac{\nabla T \boldsymbol{k} \nabla T}{T} \geq 0,
$$

are satisfied.

With the assumption that the elastic strain rate $\dot{\boldsymbol{\varepsilon}}^{e}$, and temperature rate $\dot{T}$ can have arbitrarily prescribed values and are independent of the rate of the order parameter, and applying the Coleman's exploitation, Eq. (B.3) 1 yields the two constitutive relations for the stress $\sigma$, the entropy $S$ as

$$
\sigma=\partial_{\varepsilon^{e}} \psi, \quad \text { and } S=-\partial_{T} \psi
$$

The second equation (B.5) 2 can be used to construct the thermal problem described in Eq. (8). We refer the curious reader to Refs. [40,41] for more details.

From (B.3), (B.4), and (B.5), a reduced form of the Clausius-Duhem inequality can be now expressed as

$$
\mathcal{A}_{d} \dot{d} \geq 0 \text {. }
$$

At this stage, a threshold function $F\left(\mathcal{A}_{d}\right)$ such that

$$
F\left(\mathcal{A}_{d}\right) \leq 0
$$

is introduced. Assuming the principle of maximum dissipation then requires the dissipation $\mathcal{A}_{d} \dot{d}$ to be maximum under the constraint equation (B.7). Using the method of Lagrange multipliers and the following Lagrangian is defined by

$$
\mathcal{L}=-\mathcal{A}_{d} \dot{d}+\lambda F\left(\mathcal{A}_{d}\right),
$$

yields the Kuhn-Tucker equations

$$
\frac{\partial \mathcal{L}}{\partial \mathcal{A}_{d}}=0, \lambda \geq 0, F \leq 0, \lambda F=0 .
$$

The first equality in Eq. (B.9) gives

$$
\dot{d}=\lambda \frac{\partial F\left(\mathcal{A}_{d}\right)}{\partial \mathcal{A}_{d}} \text {. }
$$

Without loss of generality, the threshold function $F\left(\mathcal{A}_{d}\right)$ is assumed in the form $F\left(\mathcal{A}_{d}\right)=\mathcal{A}_{d}$. From Eq. (B.10) and using the second inequality in Eq. (B.9), we reach

$$
\dot{d}=\lambda \geq 0
$$

For $\dot{d}>0$, and from Eqs. (B.6) and (B.11) and the third equality in Eq. (B.9), which give $F=0$, implying

$$
F=-\frac{\delta \psi}{\delta d}=-\frac{\partial \psi^{e}}{\partial d}-g_{c} \delta \gamma(d, \nabla d)=0 .
$$

With (see e.g., [19])

$$
\delta \gamma(d, \nabla d)=\frac{d}{l}-l \Delta d
$$

From Eq. (18), and Eq. (B.12) we obtain

$$
2(1-d) \psi^{e+}-g_{c} \delta \gamma(d, \nabla d)=0
$$

which defines the evolution law for the phase field $d$. As $2(1-d) \psi^{e+} \geq 0$, then

$$
\delta \gamma(d, \nabla d) \geq 0 .
$$

We can thus check that due to Eq. (B.15), the variation of crack length:

$$
\dot{\Gamma}_{l}=\int_{\Omega} \delta \gamma(d, \nabla d) \dot{d} d \Omega \geq 0,
$$

satisfying the criteria of irreversible evolution of cracks. 


\section{Appendix C. Definition of matrix form for hydro-thermo-chemical problem}

Definitions of matrix and vector forms for hydro-thermo-chemical problem:

$$
\begin{aligned}
& \mathbf{K}_{T}=\int_{\Omega} \mathbf{B}_{T}^{\mathrm{T}} \boldsymbol{k} \mathbf{B}_{T} \mathrm{~d} \Omega \\
& \mathbf{H}_{T}=\int_{\Omega} \mathbf{N}_{T}^{\mathrm{T}} \rho c \mathbf{N}_{T} \mathrm{~d} \Omega \\
& \mathbf{Q}_{c}=\int_{\partial \Omega_{c}} \mathbf{N}_{T}^{\mathrm{T}} h \mathbf{N}_{T} \mathrm{~d} S \\
& \mathbf{Q}_{\alpha}=\int_{\Omega} \mathbf{N}_{\alpha}^{\mathrm{T}} Q_{\infty} \mathbf{N}_{\alpha} \mathrm{d} \Omega \\
& \mathbf{F}_{q}=\int_{\partial \Omega_{q}} \mathbf{N}_{T}^{\mathrm{T}} \bar{q} \mathrm{~d} S \\
& \mathbf{F}_{c}=\int_{\partial \Omega_{c}} \mathbf{N}_{T}^{\mathrm{T}} h T_{a} \mathrm{~d} S \\
& \mathbf{F}_{\alpha}=\int_{\Omega} \mathbf{N}_{\alpha}^{\mathrm{T}}\left(g(d) A_{T} f(\alpha) \exp \left(-E_{a} / R T\right)\right) \mathrm{d} \Omega
\end{aligned}
$$

\section{Appendix D. Validation of the thermal/hydration model}

We additionally show here the validation of the developed approach (thermal/hydration model) by considering the early-age behavior of concrete structures, in particularly the slab strongly restrained by the supporting piles as reported in Ref. [12]. It should be noticed that, the validation of the thermal/hydration model is the missed puzzle to confirm the accuracy of our methodologies. Other issues such as the accuracy of the phase field and mechanical problems have already been demonstrated in the previous works by the present authors, see e.g., Refs. [20,34,56] for more details.

The assumption of uni-dimensional heat transfer is used, in which thermal transfer with the neighboring environment is assumed to occur across the slab thickness only. The 2-D represented model according to Ref. Faria et al. [12] is depicted in Fig. D.20. The problem is considered under the plane strain condition. The evolution of temperature and stress fields will be analyzed.

The same material parameters used in Ref. [12] are taken for the validation, which are listed in Table D.4. The investigated domain is subjected to the convection boundary conditions in the upper-end P3-P4 and lower-end P1$\mathrm{P} 2$, in which the convection coefficient is assumed to be independent of wind speed and $h_{1-2}=4.5\left[\mathrm{~W} /\left(\mathrm{m}^{2} \mathrm{~K}\right)\right]$ (convection with soil), $h_{3-4}=7.5\left[\mathrm{~W} /\left(\mathrm{m}^{2} \mathrm{~K}\right)\right]$ (convection with air). The ambient temperature is varying during the whole simulation, following the input data described in Faria et al. [12]. The isolated boundary is applied to left-end P1-P4 and right-end P2-P3. Moreover, the $x$-displacements in the left-end and right-end are fixed, while $y$-displacements are free; on the lower-end, $y$-displacements are fixed, while $x$-displacements are free. The structure is meshed using triangular elements with $h_{e}^{\max }=0.005 \mathrm{~m}$ and $h_{e}^{\min }=0.0025 \mathrm{~mm}$. The incremental time step is taken as $\Delta t=300 \mathrm{~s}$ for the first $200 \mathrm{steps}$, and then $\Delta t=600 \mathrm{~s}$ for the total of 1000 time steps.

The comparison of temperature evolution between experimental measured in Faria et al. [12] and the estimated numerical simulation by the present model at two investigated positions, e.g., IVP1 located at $(x=0.5 m, y=0.3 \mathrm{~m})$ and IVP1 located at $(x=0.5 \mathrm{~m}, y=0.05 \mathrm{~m})$, is shown in Fig. D.21(a). As expected, a good agreement between two aforementioned solutions is obtained. It is interesting to obverse from the comparison results that the numerical simulation can reproduce well the experimental results, even using a simple uni-dimensional heat transfer model.

Fig. D.21(b) shows a comparison of stress evolution obtained from new model with the one using a thermomechanical model based on finite element techniques in Faria et al. [12], herein the stress in $x$ direction at upperend (denoted [Top]) and lower-end (denoted [Bottom]) is considered. Again, the prediction of proposed model is consistent and matches well with the reference results, reflecting the accuracy of the present formulation and the correct implementation of the models. 


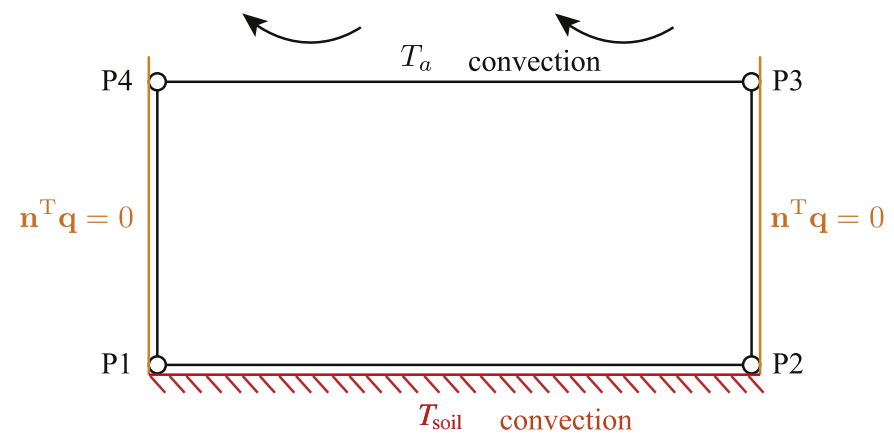

Fig. D.20. Geometry and boundary conditions of the Benchmark test, uni-dimensional heat transfer of the slab strongly restrained by the supporting piles reproduced from Faria et al. [12].

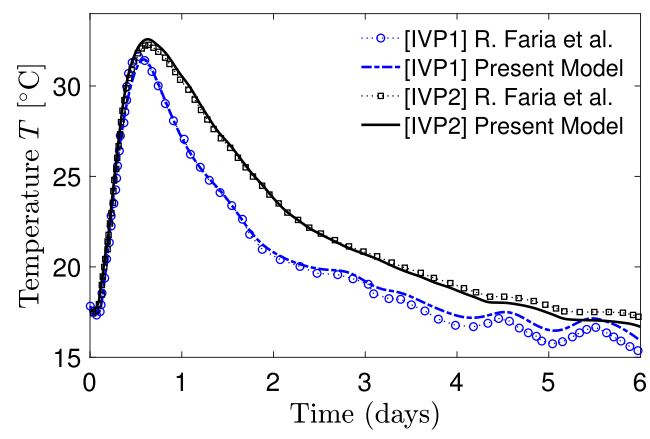

(a) Temperature evolution

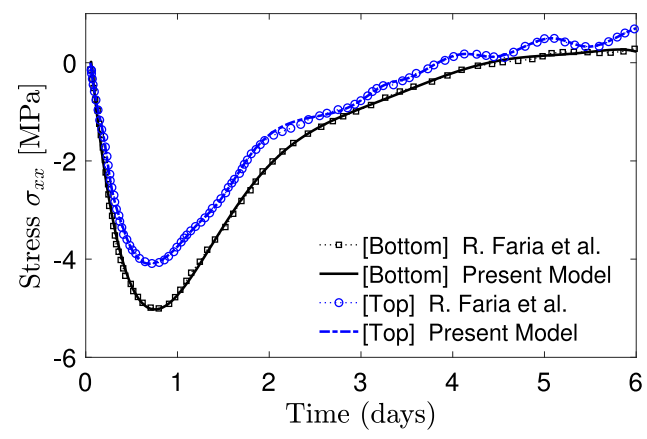

(b) Stress evolution

Fig. D.21. Validation of the numerical model proposed in the present study with the reference results reported in Faria et al. [12].

Table D.4

Material properties (Ref. [12])

\begin{tabular}{llllll}
\hline Parameter & Value & Unit & Parameter & Value & Unit \\
\hline$\rho c$ & 2400 & $\mathrm{~kJ} /\left(\mathrm{K} \mathrm{m}^{3}\right)$ & $\alpha_{a u}$ & 0.115 & - \\
$k$ & 2.6 & $\mathrm{~W} /(\mathrm{m} \mathrm{K}))$ & $\alpha_{E}$ & 0 & - \\
$Q_{\infty}$ & 119000 & $\mathrm{~kJ} / \mathrm{m}^{3}$ & $E_{a}$ & 50 & $\mathrm{~kJ} / \mathrm{mol}$ \\
$\beta$ & 10 & $\mu \mathrm{m} /(\mathrm{m} \mathrm{K})$ & $\nu$ & 0.2 & - \\
$\kappa$ & 30 & $\mu \mathrm{m} / \mathrm{m}$ & $T_{\text {soil }}$ & 17 & ${ }^{\circ} \mathrm{C}$ \\
$E_{\infty}$ & 29 & $\mathrm{GPa}$ & $A_{T}$ & 0.729 & $\mathrm{GW} / \mathrm{kg}$ \\
$h_{1-2}$ & 4.5 & $\mathrm{~W} /\left(\mathrm{m}^{2} \mathrm{~K}\right)$ & $h_{3-4}$ & 7.5 & $\mathrm{~W} /\left(\mathrm{m}^{2} \mathrm{~K}\right)$ \\
\hline
\end{tabular}

\section{References}

[1] R. de Borst, A.H. Van den Boogaard, Finite-element modeling of deformation and cracking in early-age concrete, J. Eng. Mech. 120 (12) (1994) 2519-2534.

[2] H.-W. Song, H.-J. Cho, S.-S. Park, K.-J. Byun, K. Maekawa, Early-age cracking resistance evaluation of concrete structures., Concr. Sci. Eng. 3 (10) (2001) 62-72.

[3] Y. Yuan, Z.L. Wan, Prediction of cracking within early-age concrete due to thermal, drying and creep behavior, Cement Concrete Res. 32 (7) (2002) 1053-1059.

[4] G. De Schutter, Finite element simulation of thermal cracking in massive hardening concrete elements using degree of hydration based material laws, Comput. Struct. 80 (27-30) (2002) 2035-2042.

[5] Z.P. Bažant, J.-K. Kim, S.-E. Jeon, Cohesive fracturing and stresses caused by hydration heat in massive concrete wall, J. Eng. Mech. 129 (1) (2003) 21-30.

[6] P. Grassl, H.S. Wong, N.R. Buenfeld, Influence of aggregate size and volume fraction on shrinkage induced micro-cracking of concrete and mortar, Cement Concrete Res. 40 (1) (2010) 85-93. 
[7] M. Briffaut, F. Benboudjema, J.M. Torrenti, G. Nahas, Numerical analysis of the thermal active restrained shrinkage ring test to study the early age behavior of massive concrete structures, Eng. Struct. 33 (4) (2011) 1390-1401.

[8] J. Mazars, A description of micro-and macroscale damage of concrete structures, Eng. Fract. Mech. 25 (5-6) (1986) $729-737$.

[9] M. Cervera, R. Faria, J. Oliver, T. Prato, Numerical modelling of concrete curing, regarding hydration and temperature phenomena, Comput. Struct. 80 (18-19) (2002) 1511-1521.

[10] R. Lackner, H.A. Mang, Chemoplastic material model for the simulation of early-age cracking: from the constitutive law to numerical analyses of massive concrete structures, Cement Concrete Comp. 26 (5) (2004) 551-562.

[11] V. Waller, L. d'Alora, F. Cussigh, S. Lecrux, Using the maturity method in concrete cracking control at early ages, Cement Concrete Comp. 26 (5) (2004) 589-599.

[12] R. Faria, M. Azenha, J.A. Figueiras, Modelling of concrete at early ages: application to an externally restrained slab, Cement Concrete Comp. 28 (6) (2006) 572-585.

[13] F. Benboudjema, J.-M. Torrenti, Early-age behaviour of concrete nuclear containments, Nucl. Eng. Des. 238 (10) (2008) $2495-2506$.

[14] M.N. Amin, J.-S. Kim, Y. Lee, J.-K. Kim, Simulation of the thermal stress in mass concrete using a thermal stress measuring device, Cement Concrete Res. 39 (3) (2009) 154-164.

[15] A.E. Idiart, C.M. López, I. Carol, Modeling of drying shrinkage of concrete specimens at the meso-level, Mater. Struct. 44 (2) (2011) $415-435$.

[16] G.A. Francfort, J.J. Marigo, Revisiting brittle fracture as an energy minimization problem, J. Mech. Phys. Solids 46 (8) (1998) $1319-1342$.

[17] B. Bourdin, G.A. Francfort, J.J. Marigo, Numerical experiments in revisited brittle fracture, J. Mech. Phys. Solids 48 (4) (2000) $797-826$.

[18] B. Bourdin, G.A. Francfort, J.J. Marigo, The variational approach to fracture, J. Elasticity 91 (1-3) (2008) 5-148.

[19] C. Miehe, M. Hofacker, F. Welschinger, A phase field model for rate-independent crack propagation: robust algorithmic implementation based on operator splits, Comput. Methods Appl. Mech. Engrg. 199 (2010) 2765-2778.

[20] T.T. Nguyen, J. Yvonnet, Q-Z. Zhu, M. Bornert, C. Chateau, A phase field method to simulate crack nucleation and propagation in strongly heterogeneous materials from direct imaging of their microstructure, Eng. Fract. Mech. 139 (2015) 18-39.

[21] T.T. Nguyen, J. Yvonnet, Q.-Z. Zhu, M. Bornert, C. Chateau, A phase-field method for computational modeling of interfacial damage interacting with crack propagation in realistic microstructures obtained by microtomography, Comput. Methods Appl. Mech. Engrg. 312 (2016) 567-595.

[22] T.T Nguyen, J. Yvonnet, M. Bornert, C. Chateau, F. Bilteryst, E. Steib, Large-scale simulations of quasi-brittle microcracking in realistic highly heterogeneous microstructures obtained from micro CT imaging, Extreme Mech. Lett. 17 (2017) 50-55.

[23] C. Kuhn, R. Müller, Phase field simulation of thermomechanical fracture, PAMM 9 (1) (2009) 191-192.

[24] B. Bourdin, J.-J. Marigo, C. Maurini, P. Sicsic, Morphogenesis and propagation of complex cracks induced by thermal shocks, Phys. Rev. Lett. 112 (1) (2014) 014301.

[25] P. Sicsic, J.-J. Marigo, C. Maurini, Initiation of a periodic array of cracks in the thermal shock problem: a gradient damage modeling, J. Mech. Phys. Solids 63 (2014) 256-284.

[26] C. Miehe, L.-M. Schänzel, H. Ulmer, Phase field modeling of fracture in multi-physics problems. part i. Balance of crack surface and failure criteria for brittle crack propagation in thermo-elastic solids, Comput. Methods Appl. Mech. Engrg. 294 (2015) 449-485.

[27] J.A.T. de Freitas, P.T. Cuong, R. Faria, M. Azenha, Modelling of cement hydration in concrete structures with hybrid finite elements, Finite Elem. Anal. Des. 77 (2013) 16-30.

[28] Y.R. Rashid, Analysis of reinforced concrete pressure vessels, Nucl. Eng. Des. 7 (1968) 334-344.

[29] M. Jirasek, Nonlocal models for damage and fracture: comparison of approaches, Int. J. Solids Struct. 35 (31) (1998) $4133-4145$.

[30] G.I. Barenblatt, The formation of equilibrium cracks during brittle fracture. general ideas and hypotheses. Axially-symmetric cracks, J. Appl. Math. Mech. 23 (3) (1959) 622-636.

[31] D.S. Dugdale, Yielding of steel sheets containing slits, J. Mech. Phys. Solids 8 (2) (1960) 100-104.

[32] A. Needleman, A continuum model for void nucleation by inclusion debonding, J. Appl. Mech. 54 (3) (1987) 525-531.

[33] N. Moës, J. Dolbow, T. Belytschko, A finite element method for crack growth without remeshing, Internat. J. Numer. Methods Engrg. 46 (1) (1999) 131-150.

[34] T.T. Nguyen, J. Yvonnet, M. Bornert, C. Chateau, K. Sab, R. Romani, B. Le Roy, On the choice of parameters in the phase field method for simulating crack initiation with experimental validation, Int. J. Fract. 197 (2) (2016) 213-226.

[35] K.H. Pham, K. Ravi-Chandar, C.M. Landis, Experimental validation of a phase-field model for fracture, Int. J. Fract. 205 (1) (2017) 83-101.

[36] H. Amor, J.J. Marigo, C. Maurini, Regularized formulation of the variational brittle fracture with unilateral contact: numerical experiments, J. Mech. Phys. Solids 57 (8) (2009) 1209-1229.

[37] M.J. Borden, C.V. Verhoosel, M.A. Scott, T.J.R Hughes, C.M. Landis, A phase-field description of dynamic brittle fracture, Comput. Methods Appl. Mech. Engrg. 217 (2012) 77-95.

[38] A. Schlüter, C. Kuhn, R. Müller, M. Tomut, C. Trautmann, H. Weick, C. Plate, Phase field modelling of dynamic thermal fracture in the context of irradiation damage, Contin. Mech. Thermodyn. 29 (4) (2017) 977-988.

[39] J.M. Ruiz, A.K. Schindler, R.O. Rasmussen, P.K. Nelson, G.K. Chang, Concrete temperature modeling and strength prediction using maturity concepts in the fhwa hiperpav software, in: Seventh International Conference on Concrete Pavements. The Use of Concrete in Developing Long-Lasting Pavement Solutions for the 21st CenturyInternational Society for Concrete Pavements, vol. 1, 2001.

[40] F.-J. Ulm, O. Coussy, Modeling of thermochemomechanical couplings of concrete at early ages, J. Eng. Mech. 121 (7) (1995) $785-794$.

[41] M. Cervera, J. Oliver, T. Prato, Thermo-chemo-mechanical model for concrete. i: Hydration and aging, J. Eng. Mech. 125 (9) (1999), 1018-1027.

[42] G. De Schutter, Degree of hydration based kelvin model for the basic creep of early age concrete, Mater. Struct. 32 (4) (1999) 260.

[43] L. Stefan, F. Benboudjema, J.-M. Torrenti, B. Bissonnette, Prediction of elastic properties of cement pastes at early ages, Comput. Mater. Sci. 47 (3) (2010) 775-784. 
[44] T.T. Nguyen, J. Bolivar, J. Réthoré, M-C. Baietto, M. Fregonese, A phase field method for modeling stress corrosion crack propagation in a nickel base alloy, Int. J. Solids Struct. 112 (2017) 65-82.

[45] T.T. Nguyen, J. Réthoré, J. Yvonnet, M.-C. Baietto, Multi-phase-field modeling of anisotropic crack propagation for polycrystalline materials, Comput. Mech. 60 (2017) 289-314.

[46] M.H. Protter, H.F. Weinberger, Maximum Principles in Differential Equations, Springer Science \& Business Media, 2012.

[47] C. Miehe, M. Lambrecht, Algorithms for computation of stresses and elasticity moduli in terms of seth-hill's family of generalized strain tensors, Commun. Numer. Methods Eng. 17 (2001) 337-353.

[48] M. Ambati, T. Gerasimov, L. De Lorenzis, A review on phase-field models of brittle fracture and a new fast hybrid formulation, Comput. Mech. 55 (2) (2015) 383-405.

[49] D. Schillinger, M.J. Borden, H.K. Stolarski, Isogeometric collocation for phase-field fracture models, Comput. Methods Appl. Mech. Engrg. 284 (2015) 583-610.

[50] X. Zhang, A. Krischok, C. Linder, A variational framework to model diffusion induced large plastic deformation and phase field fracture during initial two-phase lithiation of silicon electrodes, Comput. Methods Appl. Mech. Engrg. 312 (2016) 51-77.

[51] R. de Borst, S. May, J. Vignollet, A numerical assessment of phase-field models for fracture, in: Materials with Internal Structure, Springer, 2016, pp. 17-28.

[52] T. Gerasimov, L. De Lorenzis, A line search assisted monolithic approach for phase-field computing of brittle fracture, Comput. Methods Appl. Mech. Engrg. 312 (2016) 276-303.

[53] T.T. Nguyen, Modeling of complex microcracking in cement based materials by combining numerical simulations based on a phase-field method and experimental 3d imaging (Ph.D. thesis), Université Paris-Est, 2015.

[54] H.S. Wong, M. Zobel, N.R. Buenfeld, R.W. Zimmerman, Influence of the interfacial transition zone and microcracking on the diffusivity, permeability and sorptivity of cement-based materials after drying, Mag. Concr. Res. 61 (8) (2009) 571-589.

[55] F.A. Branco, P. Mendes, E. Mirambell, Heat of hydration effects in concrete structures, Mater. J. 89 (2) (1992) $139-145$.

[56] T.T. Nguyen, J. Yvonnet, M. Bornert, C. Chateau, Initiation and propagation of complex $3 \mathrm{~d}$ networks of cracks in heterogeneous quasi-brittle materials: Direct comparison between in situ testing-microCT experiments and phase field simulations, J. Mech. Phys. Solids 95 (2016) $320-350$. 